TRANSACTIONS OF THE

AMERICAN MATHEMATICAL SOCIETY

Volume 359, Number 11, November 2007, Pages 5283-5317

S 0002-9947(07)04170-0

Article electronically published on May 8, 2007

\title{
MULTIVARIABLE BOHR INEQUALITIES
}

\author{
GELU POPESCU
}

AbStract. Operator-valued multivariable Bohr type inequalities are obtained for:

(i) a class of noncommutative holomorphic functions on the open unit ball of $B(\mathcal{H})^{n}$, generalizing the analytic functions on the open unit disc;

(ii) the noncommutative disc algebra $\mathcal{A}_{n}$ and the noncommutative analytic Toeplitz algebra $F_{n}^{\infty}$;

(iii) a class of noncommutative selfadjoint harmonic functions on the open unit ball of $B(\mathcal{H})^{n}$, generalizing the real-valued harmonic functions on the open unit disc;

(iv) the Cuntz-Toeplitz algebra $C^{*}\left(S_{1}, \ldots, S_{n}\right)$, the reduced (resp. full) group $C^{*}$-algebra $C_{\text {red }}^{*}\left(\mathbb{F}_{n}\right)$ (resp. $\left.C^{*}\left(\mathbb{F}_{n}\right)\right)$ of the free group with $n$ generators;

(v) a class of analytic functions on the open unit ball of $\mathbb{C}^{n}$.

The classical Bohr inequality is shown to be a consequence of Fejér's inequality for the coefficients of positive trigonometric polynomials and Haagerup-de la Harpe inequality for nilpotent operators. Moreover, we provide an inequality which, for analytic polynomials on the open unit disc, is sharper than Bohr's inequality.

\section{INTRODUCTION}

Let $f(z):=\sum_{k=0}^{\infty} a_{k} z^{k}$ be an analytic function on the open unit disc $\mathbb{D}:=\{z \in \mathbb{C}$ : $|z|<1\}$ such that $\|f\|_{\infty} \leq 1$. Bohr's inequality [5] asserts that

$$
\sum_{k=0}^{\infty} r^{k}\left|a_{k}\right| \leq 1 \quad \text { for } 0 \leq r \leq \frac{1}{3} \text {. }
$$

Originally, the inequality was obtained for $0 \leq r \leq \frac{1}{6}$. The fact that $\frac{1}{3}$ is the best possible constant was obtained independently by M. Riesz, Schur, and Weiner. Other proofs were later obtained by Sidon [25] and Tomic [26]. Dixon [7] used Bohr's inequality in connection with the long-standing problem of characterizing Banach algebras satisfying the von Neumann inequality [28] (see also [1] and [14]). In recent years, multivariable analogues of Bohr's inequality were considered by several authors (see [1], 4], 6], and [12]). Paulsen and Singh [13] used positivity methods to obtain operator-valued generalizations of Bohr's inequality in the single variable case.

Received by the editors December 27, 2004 and, in revised form, August 17, 2005.

2000 Mathematics Subject Classification. Primary 47A20, 47A56; Secondary 47A13, 47A63.

Key words and phrases. Multivariable operator theory, Bohr's inequality, holomorphic function, harmonic function, von Neumann inequality, Poisson transform, noncommutative disc algebra, noncommutative analytic Toeplitz algebra, Fejér's inequality.

This research was supported in part by an NSF grant. 
We obtain in this paper operator-valued generalizations of Bohr's inequality in multivariable settings. Let $H_{n}$ be an $n$-dimensional complex Hilbert space with orthonormal basis $e_{1}, e_{2}, \ldots, e_{n}$, where $n \in\{1,2, \ldots\}$. We consider the full Fock space of $H_{n}$ defined by

$$
F^{2}\left(H_{n}\right):=\bigoplus_{k \geq 0} H_{n}^{\otimes k}
$$

where $H_{n}^{\otimes 0}:=\mathbb{C} 1$ and $H_{n}^{\otimes k}$ is the (Hilbert) tensor product of $k$ copies of $H_{n}$. Define the left creation operators $S_{i}: F^{2}\left(H_{n}\right) \rightarrow F^{2}\left(H_{n}\right), i=1, \ldots, n$, by

$$
S_{i} \varphi:=e_{i} \otimes \varphi, \quad \varphi \in F^{2}\left(H_{n}\right) .
$$

Let $\mathbb{F}_{n}^{+}$be the unital free semigroup on $n$ generators $g_{1}, \ldots, g_{n}$, and the identity $g_{0}$. The length of $\alpha \in \mathbb{F}_{n}^{+}$is defined by $|\alpha|:=k$ if $\alpha=g_{i_{1}} g_{i_{2}} \cdots g_{i_{k}}$, and $|\alpha|:=0$ if $\alpha=g_{0}$. If $T_{1}, \ldots, T_{n} \in B(\mathcal{H})$, the algebra of all bounded operators on a Hilbert space $\mathcal{H}$, define $T_{\alpha}:=T_{i_{1}} T_{i_{2}} \cdots T_{i_{k}}$ if $\alpha=g_{i_{1}} g_{i_{2}} \cdots g_{i_{k}}$, and $T_{g_{0}}:=I_{\mathcal{H}}$.

The noncommutative analytic Toeplitz algebra $F_{n}^{\infty}$ and its norm closed version, the noncommutative disc algebra $\mathcal{A}_{n}$, were introduced by the author [17, [18], 21] in connection with a multivariable noncommutative von Neumann inequality. $F_{n}^{\infty}$ is the algebra of left multipliers of $F^{2}\left(H_{n}\right)$ and can be identified with the weakly closed (or $w^{*}$-closed) algebra generated by the left creation operators $S_{1}, \ldots, S_{n}$ acting on $F^{2}\left(H_{n}\right)$, and the identity. The noncommutative disc algebra $\mathcal{A}_{n}$ is the norm closed algebra generated by $S_{1}, \ldots, S_{n}$, and the identity. When $n=1, F_{1}^{\infty}$ can be identified with $H^{\infty}(\mathbb{D})$, the algebra of bounded analytic functions on the open unit disc. The noncommutative analytic Toeplitz algebra $F_{n}^{\infty}$ can be viewed as a multivariable noncommutative analogue of $H^{\infty}(\mathbb{D})$. There are many analogies with the invariant subspaces of the unilateral shift on $H^{2}(\mathbb{D})$, inner-outer factorizations, analytic operators, Toeplitz operators, $H^{\infty}(\mathbb{D})$-functional calculus, bounded (resp. spectral) interpolation, etc.

In Section 1, we show that Bohr's inequality can be improved for analytic polynomials. More precisely, we prove that if $p(z):=\sum_{k=0}^{m-1} a_{k} z^{k}$ is a polynomial with $\|p\|_{\infty} \leq 1$, then

$$
\sum_{k=1}^{m-1}\left|a_{k}\right| r^{k} \leq 1 \quad \text { for } \quad 0 \leq r \leq t_{m},
$$

where $t_{m} \in(0,1]$ is the solution of the equation

$$
\sum_{k=1}^{m-1} t^{k} \cos \frac{\pi}{\left[\frac{m-1}{k}\right]+2}=\frac{1}{2},
$$

where $[x]$ is the integer part of $x$. Moreover, $\left\{t_{m}\right\}_{m=2}^{\infty}$ is a strictly decreasing sequence which converges to $\frac{1}{3}$. The above inequality is a particular case of a more general multivariable Bohr type inequality, which is obtained in Section 1, for noncommutative holomorphic functions on the open unit unit ball of $\left[B(\mathcal{X})^{n}\right]_{<1}$, i.e.,

$$
\left[B(\mathcal{X})^{n}\right]_{<1}:=\left\{\left(X_{1}, \ldots, X_{n}\right) \in B(\mathcal{X})^{n}:\left\|X_{1} X_{1}^{*}+\cdots+X_{n} X_{n}^{*}\right\|<1\right\},
$$

where $\mathcal{X}$ is an arbitrary Hilbert space. We say that $F:\left[B(\mathcal{X})^{n}\right]_{<1} \rightarrow B(\mathcal{X})$ is a universal holomorphic function on $\left[B(\mathcal{X})^{n}\right]_{<1}$ with scalar coefficients if there exists 
a sequence $\left\{a_{\alpha}\right\}_{\alpha \in \mathbb{F}_{n}^{+}} \subset \mathbb{C}$ such that

$$
F\left(X_{1}, \ldots, X_{n}\right)=\sum_{k=0}^{\infty} \sum_{|\alpha|=k} a_{\alpha} X_{\alpha}
$$

is convergent in norm for any $\left(X_{1}, \ldots, X_{n}\right) \in\left[B(\mathcal{X})^{n}\right]_{<1}$ and any Hilbert space $\mathcal{X}$. We showed in 24 that the algebra of all bounded holomorphic functions on $\left[B(\mathcal{X})^{n}\right]_{<1}$ can be identified with the noncommutative analytic Toeplitz algebra $F_{n}^{\infty}$, while the subalgebra of all holomorphic functions on $\left[B(\mathcal{X})^{n}\right]_{<1}$ and continuous on $\left[B(\mathcal{X})^{n}\right]_{\leq 1}$ can be identified with the noncommutative disc algebra $\mathcal{A}_{n}$. Many other classical results concerning the analytic functions on the open unit disc $\mathbb{D}$ were extended to this noncommutative setting in [24.

In Section 1, we also consider multivariable Bohr type inequalities for noncommutative harmonic functions on the open unit unit ball of $\left[B(\mathcal{X})^{n}\right]_{<1}$. We mention that $G$ is a selfadjoint harmonic function on $\left[B(\mathcal{X})^{n}\right]_{<1}$ if there is a universal holomorphic function $F$ on $\left[B(\mathcal{X})^{n}\right]_{<1}$ such that $G\left(X_{1}, \ldots, X_{n}\right)=\operatorname{Re} F\left(X_{1}, \ldots, X_{n}\right)$ for any $\left(X_{1}, \ldots, X_{n}\right) \in\left[B(\mathcal{X})^{n}\right]_{<1}$. As consequences, we obtain Bohr type inequalities for the Cuntz-Toeplitz algebra $C^{*}\left(S_{1}, \ldots, S_{n}\right)$, the reduced (resp. full) group $C^{*}$-algebra $C_{\text {red }}^{*}\left(\mathbb{F}_{n}\right)$ (resp. $C^{*}\left(\mathbb{F}_{n}\right)$ ) of the free group with $n$ generators. For example, given $m=2,3, \ldots, \infty$, we show that if $U_{1}, \ldots, U_{n}$ are the canonical unitaries generating $C_{r e d}^{*}\left(\mathbb{F}_{n}\right)$ and

$$
H:=\sum_{1 \leq|\alpha| \leq m-1} \bar{a}_{\alpha} U_{\alpha}^{*}+a_{0} I+\sum_{1 \leq|\alpha| \leq m-1} a_{\alpha} U_{\alpha}, \quad a_{\alpha} \in \mathbb{C},
$$

is a selfadjoint element of $C_{\text {red }}^{*}\left(\mathbb{F}_{n}\right)$ with $\|H\| \leq 1$, then

$$
\left(\sum_{|\alpha|=k}\left|a_{\alpha}\right|^{2}\right)^{1 / 2} \leq\left(1-\left|a_{0}\right|\right) \cos \frac{\pi}{\left[\frac{m-1}{k}\right]+2} \quad \text { for } k=1, \ldots, m-1
$$

and

$$
\sum_{1 \leq|\alpha| \leq m-1}\left|\bar{a}_{\alpha}\right| r_{\alpha}+\left|a_{0}\right|+\sum_{1 \leq|\alpha| \leq m-1}\left|a_{\alpha}\right| r_{\alpha} \leq 1
$$

for any $r:=\left(r_{1}, \ldots, r_{n}\right)$ with $r_{1} \geq 0, \ldots, r_{n} \geq 0$, and $\|r\|_{2} \leq t_{m}$, where $t_{m} \in(0,1]$ is the solution of the equation (0.1) if $m<\infty$ and $t_{\infty}=\frac{1}{3}$.

In Section 2, inspired by the work of Paulsen and Singh [13] in the single variable case, we obtain operator-valued multivariable Bohr type inequalities for noncommutative holomorphic functions on the open unit ball of $B(\mathcal{X})^{n}$ with coefficients in $B(\mathcal{H})$. As consequences, we obtain operator-valued Bohr inequalities for the noncommutative disc algebra $\mathcal{A}_{n}$ and the noncommutative analytic Toeplitz algebra $F_{n}^{\infty}$. In particular, we prove that if

$$
F\left(S_{1}, \ldots, S_{n}\right):=\sum_{k=0}^{\infty} \sum_{|\alpha|=k} S_{\alpha} \otimes A_{(\alpha)}, \quad A_{(\alpha)} \in B(\mathcal{H}),
$$

is in $F_{n}^{\infty} \bar{\otimes} B(\mathcal{H})$, the WOT closed algebra generated by the spatial tensor product, such that $F(0) \geq 0$ and $\operatorname{Re} F\left(S_{1}, \ldots, S_{n}\right) \leq I$, then

$$
\left\|\sum_{|\alpha|=k} A_{(\alpha)}^{*} A_{(\alpha)}\right\|^{1 / 2} \leq 2\left\|I-A_{(0)}\right\| \quad \text { for } k=1,2, \ldots,
$$


and

$$
\sum_{k=0}^{\infty}\left\|\sum_{|\alpha|=k} T_{\alpha} \otimes A_{(\alpha)}\right\| \leq\left\|A_{(0)}\right\|+\left\|I-A_{(0)}\right\|
$$

for any $n$-tuple of bounded operators $\left(T_{1}, \ldots, T_{n}\right) \in\left[B(\mathcal{K})^{n}\right]_{1 / 3}$, i.e.,

$$
\left\|T_{1} T_{1}^{*}+\cdots+T_{n} T_{n}^{*}\right\|^{1 / 2} \leq \frac{1}{3} .
$$

When $\mathcal{H}=\mathbb{C}$ and $A_{(\alpha)}=a_{\alpha} \in \mathbb{C}$, we deduce that

$$
\sum_{\alpha \in \mathbb{F}_{n}^{+}}\left|a_{\alpha}\right| r_{\alpha} \leq 1
$$

for any $r:=\left(r_{1}, \ldots, r_{n}\right)$ with $r_{1} \geq 0, \ldots, r_{n} \geq 0$, and $\|r\|_{2} \leq \frac{1}{3}$. In the single variable case $(n=1)$, we find again the classical Bohr inequality [5] and the operator-valued extension of Paulsen and Singh 13. When $m \geq 2$ and

$$
F\left(S_{1}, \ldots, S_{n}\right):=\sum_{k=0}^{m-1} \sum_{|\alpha|=k} S_{\alpha} \otimes A_{(\alpha)}
$$

is a polynomial such that $F(0) \geq 0$ and $\operatorname{Re} F\left(S_{1}, \ldots, S_{n}\right) \leq I$, then we show that

$$
w\left(A_{(\alpha)}^{*}:|\alpha|=k\right) \leq 2\left\|I-A_{(0)}\right\| \cos \frac{\pi}{\left[\frac{m-1}{k}\right]+2} \quad \text { for } \quad 1 \leq k \leq m-1
$$

and

$$
\sum_{k=0}^{m-1} r^{k} w\left(A_{(\alpha)}^{*}:|\alpha|=k\right) \leq\left\|A_{(0)}\right\|+\left\|I-A_{(0)}\right\| \quad \text { for } \quad 0 \leq r \leq t_{m}
$$

where $t_{m} \in(0,1]$ is the solution of the equation (0.1) and $w\left(X_{1}, \ldots, X_{N}\right)$ is the joint numerical radius of $\left(X_{1}, \ldots, X_{N}\right)$ (see Section 2). We remark that the above operator-valued Wiener and Bohr type inequalities are new even in the single variable case $n=1$.

In Section 3, we obtain operator-valued Bohr type inequalities for a class of noncommutative harmonic functions on the unit ball of $B(\mathcal{X})^{n}$ with coefficients in $B(\mathcal{H})$. Wiener and Bohr type inequalities are provided for the coefficients of two harmonic functions on $\left[B(\mathcal{X})^{n}\right]_{<1}$ satisfying the inequality $H_{1}\left(X_{1}, \ldots, X_{n}\right) \leq$ $H_{2}\left(X_{1}, \ldots, X_{n}\right)$. Consequently, we obtain Bohr inequalities for the spatial tensor products $C^{*}\left(S_{1}, \ldots, S_{n}\right) \otimes B(\mathcal{H}), C_{\text {red }}^{*}\left(\mathbb{F}_{n}\right) \otimes B(\mathcal{H})$, and $C^{*}\left(\mathbb{F}_{n}\right) \otimes B(\mathcal{H})$.

In Section 4 , we provide operator-valued Bohr type inequalities for a class of analytic functions on the open unit ball of $\mathbb{C}^{n}$. In the scalar case, we obtain Bohr inequalities for the elements of $H_{\mathrm{sym}}^{\infty}\left(\mathbb{B}_{n}\right)$, a Banach space of analytic functions on $\mathbb{B}_{n}$ containing all the polynomials. More precisely, given $m=2,3, \ldots, \infty$, and

$$
f\left(\lambda_{1}, \ldots, \lambda_{n}\right):=\sum_{\mathbf{p} \in \mathbb{Z}_{+}^{n},|\mathbf{p}| \leq m-1} \lambda^{\mathbf{p}} a_{\mathbf{p}}, \quad a_{\mathbf{p}} \in \mathbb{C},
$$

an analytic function in $\mathbb{B}_{n}$ such that

$$
\|f\|_{\mathrm{sym}}:=\sup _{0 \leq r<1}\left\|f_{\mathrm{sym}}\left(r S_{1}, \ldots, r S_{n}\right)\right\|<\infty
$$


(see Section 4 for the definition of the symmetrized functional calculus), we prove that

$$
\sum_{k=0}^{m-1}\left|\sum_{\mathbf{p} \in \mathbb{Z}_{+}^{n},|\mathbf{p}|=k} \lambda^{\mathbf{p}}\right| a_{\mathbf{p}}|| \leq\|f\|_{\mathrm{sym}}
$$

for any $\lambda:=\left(\lambda_{1}, \ldots, \lambda_{n}\right) \in \mathbb{B}_{n}$ with $\|\lambda\|_{2} \leq t_{m}$, where $t_{m}$ is the solution of the equation (0.1) if $m<\infty$ and $t_{\infty}=\frac{1}{3}$.

\section{Bohr INEQUalities in SEVERAL VARIABLES}

Haagerup and de la Harpe [10] proved that any bounded linear operator of norm 1 on a Hilbert space $\mathcal{H}$ such that $T^{m}=0, m \geq 2$, satisfies the inequality

$$
\omega(T) \leq \cos \frac{\pi}{m+1},
$$

where $\omega(T)$ is the numerical radius of $T$, i.e.,

$$
\omega(T):=\{\sup \{|\langle T h, h\rangle|: h \in \mathcal{H},\|h\|=1\} .
$$

They also showed that their inequality is equivalent to Fejér's inequality [8] for positive trigonometric polynomials of the form

$$
f\left(e^{i \theta}\right):=\sum_{k=-m+1}^{m-1} a_{k} e^{i k \theta}, \quad a_{k} \in \mathbb{C},
$$

which asserts that

$$
\left|a_{1}\right| \leq a_{0} \cos \frac{\pi}{m+1} .
$$

In 23, we obtained multivariable generalizations of the Haagerup-de la Harpe inequality and multivariable noncommutative (resp. commutative) analogues of classical inequalities (Fejér [8], Egerváry-Százs [9]) for the coefficients of positive trigonometric polynomials. In particular, we showed that if

$$
f=\sum_{1 \leq|\alpha| \leq m-1} \bar{a}_{\alpha} S_{\alpha}^{*}+a_{0} I+\sum_{1 \leq|\alpha| \leq m-1} a_{\alpha} S_{\alpha}
$$

is a positive polynomial in $C^{*}\left(S_{1}, \ldots, S_{n}\right)$, then

$$
\left(\sum_{|\alpha|=k}\left|a_{\alpha}\right|^{2}\right)^{1 / 2} \leq a_{0} \cos \frac{\pi}{\left[\frac{m-1}{k}\right]+2}
$$

for $1 \leq k \leq m-1$, where $[x]$ is the integer part of $x$.

For $r \geq 0$, we define

$$
\left[B(\mathcal{K})^{n}\right]_{r}:=\left\{\left(T_{1}, \ldots, T_{n}\right):\left\|T_{1} T_{1}^{*}+\cdots+T_{n} T_{n}^{*}\right\|^{1 / 2} \leq r\right\} .
$$

In what follows we obtain a Bohr type inequality for analytic polynomials in the Cuntz-Toeplitz algebra $C^{*}\left(S_{1}, \ldots, S_{n}\right)$.

Theorem 1.1. Let $m \geq 2$ and let $p\left(S_{1}, \ldots, S_{n}\right):=\sum_{|\alpha| \leq m-1} a_{\alpha} S_{\alpha}$ be a polynomial such that $p(0) \geq 0$ and $\operatorname{Re} p\left(S_{1}, \ldots, S_{n}\right) \leq I$. Then

$$
\sum_{k=0}^{m-1}\left\|\sum_{|\alpha|=k}\left|a_{\alpha}\right| T_{\alpha}\right\| \leq 1
$$


for any $\left(T_{1}, \ldots, T_{n}\right) \in\left[B(\mathcal{K})^{n}\right]_{t_{m}}$, where $t_{m} \in(0,1]$ is the positive solution of the equation

$$
\sum_{k=1}^{m-1} t^{k} \cos \frac{\pi}{\left[\frac{m-1}{k}\right]+2}=\frac{1}{2},
$$

where $[x]$ is the integer part of $x$. Moreover, $\left\{t_{m}\right\}$ is a strictly decreasing sequence which converges to $\frac{1}{3}$. In particular,

$$
\sum_{|\alpha| \leq m-1}\left|a_{\alpha}\right| r_{\alpha} \leq 1
$$

for any $r:=\left(r_{1}, \ldots, r_{n}\right)$ with $r_{1} \geq 0, \ldots, r_{n} \geq 0$, and $\|r\|_{2} \leq t_{m}$.

Proof. The conditions $p(0) \geq 0$ and $\operatorname{Re} p\left(S_{1}, \ldots, S_{n}\right) \leq I$ imply

$$
\sum_{1 \leq|\alpha| \leq m-1}-\bar{a}_{\alpha} S_{\alpha}^{*}+2\left(1-a_{0}\right) I+\sum_{1 \leq|\alpha| \leq m-1}-a_{\alpha} S_{\alpha} \geq 0 .
$$

According to the inequality (1.1), we have

$$
\left(\sum_{|\alpha|=k}\left|a_{\alpha}\right|^{2}\right)^{1 / 2} \leq 2\left(1-a_{0}\right) \cos \frac{\pi}{\left[\frac{m-1}{k}\right]+2}
$$

for $1 \leq k \leq m-1$. Let $\left(T_{1}, \ldots, T_{n}\right) \in\left[B(\mathcal{K})^{n}\right]_{r}$, where $0 \leq r \leq t_{m}$ and $t_{m}$ is the positive solution of equation (1.2). Using the noncommutative von Neumann inequality [17] for the row contraction $\left[r^{-1} T_{1}, \ldots, r^{-1} T_{n}\right]$ and inequality (1.3), we deduce that

$$
\begin{aligned}
\sum_{k=0}^{m-1}\left\|\sum_{|\alpha|=k}\left|a_{\alpha}\right| T_{\alpha}\right\| & \leq \sum_{k=0}^{m-1}\left\|r^{k} \sum_{|\alpha|=k}\left|a_{\alpha}\right| S_{\alpha}\right\| \\
& =\sum_{k=0}^{m-1} r^{k}\left(\sum_{|\alpha|=k}\left|a_{\alpha}\right|^{2}\right)^{1 / 2} \\
& \leq a_{0}+2\left(1-a_{0}\right) \sum_{k=1}^{m-1} r^{k} \cos \frac{\pi}{\left[\frac{m-1}{k}\right]+2} \\
& \leq a_{0}+2\left(1-a_{0}\right) \sum_{k=1}^{m-1} t_{m}^{k} \cos \frac{\pi}{\left[\frac{m-1}{k}\right]+2} \\
& =a_{0}+\left(1-a_{0}\right)=1 .
\end{aligned}
$$

For each $m \geq 2$, define the function $f_{m}:[0,1] \rightarrow[0, \infty)$ by setting

$$
f_{m}(t):=\sum_{k=1}^{m-1} t^{k} \cos \frac{\pi}{\left[\frac{m-1}{k}\right]+2} .
$$

Notice that $f_{m}(0)=0$ and $f_{m}(1)>\cos \frac{\pi}{3}=\frac{1}{2}$. Since $f_{m}$ is strictly increasing and continuous, the equation $f_{m}(t)=\frac{1}{2}$ has a unique solution $t_{m} \in(0,1]$. On the other hand, notice that $f_{m}(t)<f_{m+1}(t)<f(t)$ for any $t \in[0,1)$ and $m \geq 2$, where 
$f(t)=\sum_{k=1}^{\infty} t^{k}$. Since $f\left(\frac{1}{3}\right)=\frac{1}{2}$, it is clear that $t_{m}>\frac{1}{3}$ and the sequence $\left\{t_{m}\right\}_{m=2}^{\infty}$ is strictly decreasing.

A closer look at the the sequence $\left\{f_{m}\right\}$ reveals that it is uniformly convergent to $f$ on any interval $[0, \delta]$ with $0<\delta<1$. Notice also that $f_{m}^{\prime}(t) \geq \cos \frac{\pi}{m+1} \geq \frac{1}{2}$ for any $m \geq 2$ and $t \in[0,1]$. Applying the Lagrange mean value theorem to the function $f_{m}$ on the interval $\left[\frac{1}{3}, t_{m}\right]$, we find $\xi \in\left(\frac{1}{3}, t_{m}\right)$ such that

$$
\begin{aligned}
\frac{1}{2}-f_{m}\left(\frac{1}{3}\right) & =f_{m}\left(t_{m}\right)-f_{m}\left(\frac{1}{3}\right)=f_{m}^{\prime}\left(\xi_{m}\right)\left(t_{m}-\frac{1}{3}\right) \\
& \geq \frac{1}{2}\left(t_{m}-\frac{1}{3}\right)>0 .
\end{aligned}
$$

Hence, and since $f_{m}\left(\frac{1}{3}\right) \rightarrow f\left(\frac{1}{3}\right)=\frac{1}{2}$, as $m \rightarrow \infty$, we deduce that $t_{m} \rightarrow \frac{1}{3}$. The proof is complete.

Simple computations reveal that $t_{2}=1$ and $t_{3}=\frac{\sqrt{6}-\sqrt{2}}{2}$. We also remark that if $\left\|p\left(S_{1}, \ldots, S_{n}\right)\right\| \leq 1$, then $\operatorname{Re} p\left(S_{1}, \ldots, S_{n}\right) \leq 1$ and, consequently, Theorem 1.1 holds.

Lemma 1.2. Let $f(z)=\sum_{k=0}^{\infty} a_{k} z^{k}$ be an analytic function on the open unit disc. Then $\operatorname{Re} f(z) \leq 1$ for any $z \in \mathbb{D}$ if and only if $\operatorname{Re} f(r S) \leq 1$ for $0 \leq r<1$, where $S$ is the unilateral shift. Moreover, if $p$ is an analytic polynomial, then $\operatorname{Re} p(z) \leq 1$ if and only if $\operatorname{Re} p(S) \leq I$.

Proof. Assume that $\operatorname{Re} f(z) \leq 1$ for any $z \in \mathbb{D}$. Notice that, for each $r \in[0,1)$, the function $f_{r}\left(e^{i t}\right):=\sum_{k=0}^{\infty} r^{k} a_{k} e^{i k t}$ is in the Hardy space $H^{\infty}(\mathbb{T})$. Moreover, $f_{r}(S):=$ $\sum_{k=0}^{\infty} r^{k} a_{k} S^{k}$ is convergent in the operator norm. For every $h\left(e^{i t}\right) \in H^{2}(\mathbb{T})$, we have

$$
\begin{aligned}
&\left\langle\left[2 I-\left(f_{r}(S)+f_{r}(S)^{*}\right)\right]\right.\left.h\left(e^{i t}\right), h\left(e^{i t}\right)\right\rangle_{H^{2}(\mathbb{T})} \\
&=\frac{1}{2 \pi} \int_{-\pi}^{\pi}\left[2-\left(f_{r}\left(e^{i t}\right)+\overline{f_{r}\left(e^{i t}\right)}\right)\right]\left|h\left(e^{i t}\right)\right|^{2} d t \geq 0 .
\end{aligned}
$$

Therefore, we have $\operatorname{Re} f(r S) \leq 1$ for $0 \leq r<1$.

Conversely, assume that $f_{r}(S)^{*}+f_{r}(S) \leq 2 I$ for any $r \in[0,1)$. Using the Poisson transform of [22] associated with the contraction $T:=z I$, where $z \in \mathbb{D}$, we deduce that $\overline{f_{r}(z)}+f_{r}(z) \leq 2$ for any $z \in \mathbb{D}$ and $r \in[0,1)$. Therefore, Re $f(z) \leq 1$ for any $z \in \mathbb{D}$. The last part of the lemma is now obvious. The proof is complete.

When $n=1$, inequality (1.1), Theorem 1.1, and Lemma 1.2 imply the following result.

Corollary 1.3. Let $m \geq 2$ and let $p(z)=\sum_{k=0}^{m-1} a_{k} z^{k}$ be an analytic polynomial on the open unit disc such that $p(0) \geq 0$ and $\operatorname{Re} p(z) \leq 1$ for $z \in \mathbb{D}$. Then

$$
\left|a_{k}\right| \leq 2\left(1-a_{0}\right) \cos \frac{\pi}{\left[\frac{m-1}{k}\right]+2} \quad \text { for } 1 \leq k \leq m-1
$$


and

$$
\sum_{k=0}^{m-1}\left|a_{k}\right| r^{k} \leq 1 \quad \text { for } \quad 0 \leq r \leq t_{m}
$$

where $t_{m}$ is the solution of equation (1.2).

Notice that if $p$ is a polynomial with $\|p\|_{\infty} \leq 1$, then inequality (1.4) holds and is sharper than Bohr's inequality, when restricted to polynomials.

Now we can prove the following multivariable Bohr type inequality for noncommutative holomorphic functions on the unit open unit ball of $\left[B(\mathcal{X})^{n}\right]_{<1}$.

Theorem 1.4. Let $f\left(X_{1}, \ldots, X_{n}\right):=\sum_{k=0}^{\infty} \sum_{|\alpha|=k} a_{\alpha} X_{\alpha}, \quad a_{\alpha} \in \mathbb{C}$, be a holomorphic function on $\left[B(\mathcal{X})^{n}\right]_{<1}$ such that $f(0) \geq 0$ and

$$
\operatorname{Re} f\left(X_{1}, \ldots, X_{n}\right) \leq I \quad \text { for any }\left(X_{1}, \ldots, X_{n}\right) \in\left[B(\mathcal{X})^{n}\right]_{<1} \text {. }
$$

Then

$$
\left(\sum_{|\alpha|=k}\left|a_{\alpha}\right|^{2}\right)^{1 / 2} \leq 2\left(1-a_{0}\right) \quad \text { for } k=1,2, \ldots,
$$

and

$$
\sum_{k=0}^{\infty}\left\|\sum_{|\alpha|=k}\left|a_{\alpha}\right| T_{\alpha}\right\| \leq 1
$$

for any $\left(T_{1}, \ldots, T_{n}\right) \in\left[B(\mathcal{K})^{n}\right]_{1 / 3}$. In particular,

$$
\sum_{\alpha \in \mathbb{F}_{n}^{+}}\left|a_{\alpha}\right| r_{\alpha} \leq 1
$$

for any $r:=\left(r_{1}, \ldots, r_{n}\right)$ with $r_{1} \geq 0, \ldots, r_{n} \geq 0$ and $\|r\|_{2} \leq \frac{1}{3}$.

Proof. Since $f$ is holomorphic function on $\left[B(\mathcal{X})^{n}\right]_{<1}$ we have that the series $\sum_{k=0}^{\infty} r^{k}\left(\sum_{|\alpha|=k}\left|a_{\alpha}\right|^{2}\right)^{1 / 2}$ is convergent for any $r \in[0,1)$. Using the noncommutative Poisson transforms of [22], one can easily prove that $\operatorname{Re} f\left(X_{1}, \ldots, X_{n}\right) \leq I$ for any $\left(X_{1}, \ldots, X_{n}\right) \in\left[B(\mathcal{X})^{n}\right]_{<1}$ if and only if $\operatorname{Re} f\left(r S_{1}, \ldots, r S_{n}\right) \leq I$ for any $r \in[0,1)$. Therefore, we have

$$
\sum_{k=1}^{\infty} \sum_{|\alpha|=k}-r^{|\alpha|} \bar{a}_{\alpha} S_{\alpha}^{*}+2\left(1-a_{0}\right) I+\sum_{k=1}^{\infty} \sum_{|\alpha|=k}-r^{|\alpha|} a_{\alpha} S_{\alpha} \geq 0 .
$$

For each $m=2,3, \ldots$, and $r \in[0,1)$, denote

$$
M_{m}(r):=\sum_{k \geq m} r^{k}\left(\sum_{|\alpha|=k}\left|a_{\alpha}\right|^{2}\right)^{1 / 2} .
$$

Notice that, for each $r \in[0,1), M_{m}(r) \rightarrow 0$, as $m \rightarrow \infty$. On the other hand, the inequality (1.6) implies

$$
\sum_{1 \leq|\alpha| \leq m-1}-r^{|\alpha|} \bar{a}_{\alpha} S_{\alpha}^{*}+2\left(1-a_{0}+M_{m}(r)\right) I+\sum_{1 \leq|\alpha| \leq m-1}-r^{|\alpha|} a_{\alpha} S_{\alpha} \geq 0
$$


for any $r \in[0,1)$. According to the inequality (1.1), we obtain

$$
r^{k}\left(\sum_{|\alpha|=k}\left|a_{\alpha}\right|^{2}\right)^{1 / 2} \leq 2\left(1-a_{0}+M_{m}(r)\right) \cos \frac{\pi}{\left[\frac{m-1}{k}\right]+2}
$$

for $1 \leq k \leq m-1$ and any $r \in[0,1)$. Taking $m \rightarrow \infty$ and then $r \rightarrow 1$, we get the inequality (1.5). Hence, and using the noncommutative von Neumann inequality if $\left(T_{1}, \ldots, T_{n}\right) \in\left[B(\mathcal{K})^{n}\right]_{t}$ and $0 \leq t \leq \frac{1}{3}$, we obtain

$$
\begin{aligned}
\sum_{k=0}^{\infty}\left\|\sum_{|\alpha|=k}\left|a_{\alpha}\right| T_{\alpha}\right\| & \leq \sum_{k=0}^{\infty} t^{k}\left\|\sum_{|\alpha|=k}\left|a_{\alpha}\right| S_{\alpha}\right\| \\
& =\sum_{k=0}^{\infty} t^{k}\left(\sum_{|\alpha|=k}\left|a_{\alpha}\right|^{2}\right)^{1 / 2} \\
& \leq\left|a_{0}\right|+2\left|1-a_{0}\right| \frac{t}{1-t} \\
& \leq\left|a_{0}\right|+\left|1-a_{0}\right|=1
\end{aligned}
$$

for $0 \leq t \leq \frac{1}{3}$. This completes the proof.

Corollary $1.5([12])$. Let $f(z)=\sum_{k=0}^{\infty} a_{k} z^{k}$ be an analytic function on the open unit disc such that $f(0) \geq 0$ and $\operatorname{Re} f(z) \leq 1$ for $z \in \mathbb{D}$. Then

$$
\sum_{k=0}^{\infty}\left|a_{k}\right| r^{k} \leq 1 \quad \text { for } 0 \leq r \leq \frac{1}{3}
$$

Proof. Since $\limsup _{k \rightarrow \infty}\left|a_{k}\right|^{1 / k} \leq 1$, it is clear that $\sum_{n=0}^{\infty} r^{n} a_{n} S^{n}$ is norm convergent for any $r \in[0,1)$. Now the result follows from Lemma 1.2 and Theorem 1.4 .

Another consequence of Theorem 1.4 is the following Bohr inequality for the noncommutative analytic Toeplitz algebra.

Corollary 1.6. If $f\left(S_{1}, \ldots, S_{n}\right):=\sum_{\alpha \in \mathbb{F}_{n}^{+}} a_{\alpha} S_{\alpha}$ is in the noncommutative analytic Toeplitz algebra $F_{n}^{\infty}$, then

$$
\sum_{k=0}^{\infty}\left\|\sum_{|\alpha|=k}\left|a_{\alpha}\right| T_{\alpha}\right\| \leq\left\|f\left(S_{1}, \ldots, S_{n}\right)\right\|
$$

for any $\left(T_{1}, \ldots, T_{n}\right) \in\left[B(\mathcal{K})^{n}\right]_{1 / 3}$. In particular,

$$
\sum_{\alpha \in \mathbb{F}_{n}^{+}}\left|a_{\alpha}\right| r_{\alpha} \leq\left\|f\left(S_{1}, \ldots, S_{n}\right)\right\|
$$

for any $r:=\left(r_{1}, \ldots, r_{n}\right)$ with $r_{1} \geq 0, \ldots, r_{n} \geq 0$ and $\|r\|_{2} \leq \frac{1}{3}$.

Proof. It follows from Theorem 1.4 if we assume that $\left\|f\left(S_{1}, \ldots, S_{n}\right)\right\|=1$ and notice that, since $f\left(S_{1}, \ldots, S_{n}\right) \in F_{n}^{\infty}$, the series $\sum_{k=0}^{\infty}\left\|\sum_{|\alpha|=k} r^{|\alpha|} a_{\alpha} S_{\alpha}\right\|$ is convergent. 
We remark that Corollary [1.6 was obtained in [12, using different methods. When $n=1$, Corollary 1.6 and Lemma 1.2 imply the classical Bohr inequality.

Corollary 1.7 (Bohr's inequality). If $f(z)=\sum_{k=0}^{\infty} a_{k} z^{k}$ is a bounded analytic function on the open unit disc, then

$$
\sum_{k=0}^{\infty}\left|a_{k}\right| r^{k} \leq\|f\|_{\infty} \quad \text { for } \quad 0 \leq r \leq \frac{1}{3}
$$

We remark that, in the particular case $n=1$, the proofs of Theorem 1.1, Theorem 1.4. Corollary 1.6. and Corollary 1.7 show that Fejér's inequality for the coefficients of positive trigonometric polynomials implies Bohr's inequality for bounded analytic functions on the open unit disc. Here, we should add that Fejér's inequality is equivalent to the Haagerup-de la Harpe inequality, which implies the EgervärySzász inequality (i.e., inequality (1.1) in the particular case when $n=1$ and $2 \leq$ $k \leq m-1)$ (see 23 ).

In what follows we obtain Bohr type inequalities for a class of selfadjoint polynomials in the Cuntz-Toeplitz $C^{*}$-algebra $C^{*}\left(S_{1}, \ldots, S_{n}\right)$.

Theorem 1.8. Let $m \geq 2$ and let

$$
H\left(S_{1}, \ldots, S_{n}\right):=\sum_{k=1}^{m-1} \sum_{|\alpha|=k} \bar{a}_{\alpha} S_{\alpha}^{*}+a_{0} I+\sum_{k=1}^{m-1} \sum_{|\alpha|=k} a_{\alpha} S_{\alpha}, \quad a_{\alpha} \in \mathbb{C},
$$

be a selfadjoint element of $C^{*}\left(S_{1}, \ldots, S_{n}\right)$ such that $\left\|H\left(S_{1}, \ldots, S_{n}\right)\right\| \leq 1$.

Then the following statements hold:

(i) $\left(\sum_{|\alpha|=k}\left|a_{\alpha}\right|^{2}\right)^{1 / 2} \leq\left(1-\left|a_{0}\right|\right) \cos \frac{\pi}{\left[\frac{m-1}{k}\right]}$ for $1 \leq k \leq m-1$, where $[x]$ is the integer part of $x$.

(ii) $\sum_{k=0}^{m-1}\left\|\sum_{|\alpha|=k}\left|a_{\alpha}\right| T_{\alpha}\right\| \leq 1 \quad$ for any $\left[T_{1}, \ldots, T_{n}\right] \in\left[B(\mathcal{H})^{n}\right]_{\gamma_{m}}$, where $\gamma_{m} \in$ $(0,1]$ is the solution of the equation

$$
\sum_{k=1}^{m-1} t^{k} \cos \frac{\pi}{\left[\frac{m-1}{k}\right]+2}=1 .
$$

Moreover, $\left\{\gamma_{m}\right\}$ is a strictly decreasing sequence which converges to $\frac{1}{2}$.

(iii) $\sum_{k=1}^{m-1}\left\|\sum_{|\alpha|=k}\left|\bar{a}_{\alpha}\right| T_{\alpha}^{*}\right\|+\left|a_{0}\right|+\sum_{k=1}^{m-1}\left\|\sum_{|\alpha|=k}\left|a_{\alpha}\right| T_{\alpha}\right\| \leq 1$ for any $\left[T_{1}, \ldots, T_{n}\right] \in$ $\left[B(\mathcal{H})^{n}\right]_{t_{m}}$, where $t_{m} \in(0,1]$ is the solution of the equation

$$
\sum_{k=1}^{m-1} t^{k} \cos \frac{\pi}{\left[\frac{m-1}{k}\right]+2}=\frac{1}{2} .
$$


Moreover, $\left\{t_{m}\right\}$ is a strictly decreasing sequence which converges to $\frac{1}{3}$.

In particular, if $r_{1}, \ldots, r_{n} \geq 0$, then

(iv) $\sum_{k=0}^{m-1}\left(\sum_{|\alpha|=k}\left|a_{\alpha}\right| r_{\alpha}\right) \leq 1$ if $\left\|\left(r_{1}, \ldots, r_{n}\right)\right\|_{2} \leq \gamma_{m}$.

(v) $\left|a_{0}\right|+2 \sum_{k=1}^{m-1}\left(\sum_{|\alpha|=k}\left|a_{\alpha}\right| r_{\alpha}\right) \leq 1$ if $\left\|\left(r_{1}, \ldots, r_{n}\right)\right\|_{2} \leq t_{m}$.

Proof. Notice that we can assume that $a_{0}>0$ and

$$
\sum_{k=1}^{m-1} \sum_{|\alpha|=k}-\bar{a}_{\alpha} S_{\alpha}^{*}+\left(1-a_{0}\right) I+\sum_{|\alpha|=k}-a_{\alpha} S_{\alpha} \geq 0 .
$$

Using inequality (1.1), we deduce (i). The rest of the proof is similar to that of Theorem 1.1. We shall omit it.

Let $\mathbb{F}_{n}$ be the free group with generators $g_{1}, \ldots, g_{n}$, and let $\ell^{2}\left(\mathbb{F}_{n}\right)$ be the Hilbert space defined by

$$
\ell^{2}\left(\mathbb{F}_{n}\right):=\left\{f: \mathbb{F}_{n} \rightarrow \mathbb{C}: \sum_{\sigma \in \mathbb{F}_{n}}|f(\sigma)|^{2}<\infty\right\} .
$$

The canonical basis of $\ell^{2}\left(\mathbb{F}_{n}\right)$ is $\left\{\xi_{\sigma}\right\}_{\sigma \in \mathbb{F}_{n}}$, where $\xi_{\sigma}(t)=1$ if $t=\sigma$ and $\xi_{\sigma}(t)=0$ otherwise. For each $i=1, \ldots, n$, let $U_{i} \in B\left(\ell^{2}\left(\mathbb{F}_{n}\right)\right)$ be the unitary operator defined by

$$
U_{i}\left(\sum_{\sigma \in \mathbb{F}_{n}} a_{\sigma} \xi_{\sigma}\right):=\sum_{\sigma \in \mathbb{F}_{n}} a_{\sigma} \xi_{g_{i} \sigma}, \quad\left(\sum_{\sigma \in \mathbb{F}_{n}}\left|a_{\sigma}\right|^{2}<\infty\right) .
$$

The reduced group $C^{*}$-algebra $C_{\text {red }}^{*}\left(\mathbb{F}_{n}\right)$ is the $C^{*}$-algebra generated by $U_{1}, \ldots, U_{n}$.

Corollary 1.9. Let

$$
H\left(U_{1}, \ldots, U_{n}\right):=\sum_{k=1}^{m-1} \sum_{|\alpha|=k} \bar{a}_{\alpha} U_{\alpha}^{*}+a_{0} I+\sum_{k=1}^{m-1} \sum_{|\alpha|=k} a_{\alpha} U_{\alpha}, \quad a_{\alpha} \in \mathbb{C},
$$

be a selfadjoint element of $C_{\mathrm{red}}^{*}\left(\mathbb{F}_{n}\right)$ with $\left\|H\left(U_{1}, \ldots, U_{n}\right)\right\| \leq 1$. Then the conclusion of Theorem 1.8 holds.

Proof. Notice that the Hilbert space $\ell^{2}\left(\mathbb{F}_{n}^{+}\right)$can be seen as a subspace of $\ell^{2}\left(\mathbb{F}_{n}\right)$ and the full Fock space $F^{2}\left(H_{n}\right)$ can be naturally identified to $\ell^{2}\left(\mathbb{F}_{n}^{+}\right)$. Under this identification, we have $\left.U_{i}\right|_{F^{2}\left(H_{n}\right)}=S_{i}, i=1, \ldots, n$, where $S_{1}, \ldots, S_{n}$ are the left creation operators. Consequently, we have

$$
H\left(S_{1}, \ldots, S_{n}\right)=P_{\ell^{2}\left(\mathbb{F}_{n}^{+}\right)} H\left(U_{1}, \ldots, U_{n}\right) \mid \ell^{2}\left(\mathbb{F}_{n}^{+}\right) .
$$

Since $\left\|H\left(U_{1}, \ldots, U_{n}\right)\right\| \leq 1$, we have $\left\|H\left(S_{1}, \ldots, S_{n}\right)\right\| \leq 1$. Now applying Theorem 1.8, the result follows.

We recall that the full group $C^{*}$-algebra $C^{*}\left(\mathbb{F}_{n}\right)$ is generated by an $n$-tuple of universal unitaries $\mathbf{U}_{1}, \ldots, \mathbf{U}_{n}$. We remark that a result similar to Corollary 1.9 holds for $C^{*}\left(\mathbb{F}_{n}\right)$. Indeed, assume that $\left\|H\left(\mathbf{U}_{1}, \ldots, \mathbf{U}_{n}\right)\right\| \leq 1$. Due to the universal property of the unitaries $\mathbf{U}_{1}, \ldots, \mathbf{U}_{n}$, there is a $*$-representation $\pi$ of $C^{*}\left(\mathbf{U}_{1}, \ldots, \mathbf{U}_{n}\right)$ onto $C^{*}\left(U_{1}, \ldots, U_{n}\right)$ such that $\pi\left(\mathbf{U}_{i}\right)=U_{i}, i=1, \ldots, n$. Therefore, $H\left(U_{1}, \ldots, U_{n}\right)=\pi\left(H\left(\mathbf{U}_{1}, \ldots, \mathbf{U}_{n}\right)\right) \leq I$. Now applying Corollary [1.9, the result follows. 
The following result is a multivariable Bohr inequality for selfadjoint harmonic functions on the open unit ball of $\left[B(\mathcal{X})^{n}\right]_{<1}$.

Theorem 1.10. Let

$$
H\left(X_{1}, \ldots, X_{n}\right):=\sum_{k=1}^{\infty} \sum_{|\alpha|=k} \bar{a}_{\alpha} X_{\alpha}^{*}+a_{0} I+\sum_{k=1}^{\infty} \sum_{|\alpha|=k} a_{\alpha} X_{\alpha}, \quad a_{\alpha} \in \mathbb{C},
$$

be a selfadjoint harmonic function on $\left[B(\mathcal{X})^{n}\right]_{<1}$ such that $\left\|H\left(X_{1}, \ldots, X_{n}\right)\right\| \leq 1$ for any $\left(X_{1}, \ldots, X_{n}\right) \in\left[B(\mathcal{X})^{n}\right]_{<1}$.

Then

(i) $\left(\sum_{|\alpha|=k}\left|a_{\alpha}\right|^{2}\right)^{1 / 2} \leq 1-\left|a_{0}\right|$ for any $k=1,2, \ldots$;

(ii) $\sum_{k=0}^{\infty}\left\|\sum_{|\alpha|=k}\left|a_{\alpha}\right| T_{\alpha}\right\| \leq 1$ for any $\left[T_{1}, \ldots, T_{n}\right] \in\left[B(\mathcal{H})^{n}\right]_{1 / 2}$;

(iii) $\sum_{k=1}^{\infty}\left\|\sum_{|\alpha|=k}\left|\bar{a}_{\alpha}\right| T_{\alpha}^{*}\right\|+\left|a_{0}\right|+\sum_{k=1}^{\infty}\left\|\sum_{|\alpha|=k}\left|a_{\alpha}\right| T_{\alpha}\right\| \leq 1 \quad$ for any $\left[T_{1}, \ldots, T_{n}\right] \in$ $\left[B(\mathcal{H})^{n}\right]_{1 / 3}$.

In particular, if $r_{1}, \ldots, r_{n} \geq 0$, then

(iv) $\sum_{k=0}^{\infty}\left(\sum_{|\alpha|=k}\left|a_{\alpha}\right| r_{\alpha}\right) \leq 1$ if $\left\|\left(r_{1}, \ldots, r_{n}\right)\right\|_{2} \leq \frac{1}{2}$;

(v) $\left|a_{0}\right|+2 \sum_{k=1}^{\infty}\left(\sum_{|\alpha|=k}\left|a_{\alpha}\right| r_{\alpha}\right) \leq 1$ if $\left\|\left(r_{1}, \ldots, r_{n}\right)\right\|_{2} \leq \frac{1}{3}$.

Proof. Since $H\left(X_{1}, \ldots, X_{n}\right)$ is selfadjoint and $\left\|H\left(X_{1}, \ldots, X_{n}\right)\right\| \leq 1$, we can assume that $a_{0} \geq 0$. Therefore, we have

$$
\sum_{k=1}^{\infty} \sum_{|\alpha|=k} r^{|\alpha|} \bar{b}_{\alpha} S_{\alpha}^{*}+b_{0} I+\sum_{k=1}^{\infty} \sum_{|\alpha|=k} r^{|\alpha|} b_{\alpha} S_{\alpha} \geq 0, \quad r \in[0,1),
$$

where $b_{0}:=1-a_{0}$ and $b_{\alpha}:=-a_{\alpha}$ if $\alpha \in \mathbb{F}_{n}^{+},|\alpha| \geq 1$. As in the proof of Theorem 1.4. we deduce the inequality (i). Hence, it is easy to see that if $r \leq \frac{1}{2}$, then

$$
\sum_{k=0}^{\infty} r^{k}\left(\sum_{|\alpha|=k}\left|a_{\alpha}\right|^{2}\right)^{1 / 2} \leq a_{0}+\frac{r}{1-r}\left(1-a_{0}\right) \leq 1 .
$$

Let $\left(T_{1}, \ldots, T_{n}\right) \in\left[B(\mathcal{H})^{n}\right]_{r}$ and assume that $0<r \leq \frac{1}{2}$. Using the noncommutative von Neumann inequality [17] for the row contraction $\left[\frac{1}{r} T_{1}, \ldots, \frac{1}{r} T_{n}\right]$ and inequality (1.9), we get

$$
\begin{aligned}
\sum_{k=0}^{\infty}\left\|\sum_{|\alpha|=k}\left|a_{\alpha}\right| T_{\alpha}\right\| & \leq \sum_{k=0}^{\infty} r^{k}\left\|\sum_{|\alpha|=k}\left|a_{\alpha}\right| S_{\alpha}\right\| \\
& =\sum_{k=0}^{\infty} r^{k}\left(\sum_{|\alpha|=k}\left|a_{\alpha}\right|^{2}\right)^{1 / 2} \leq 1,
\end{aligned}
$$


which proves the inequality (ii). To prove (iii), notice that

$$
\sum_{k=1}^{\infty} r^{k}\left(\sum_{|\alpha|=k}\left|\bar{a}_{\alpha}\right|^{2}\right)^{1 / 2}+\sum_{k=0}^{\infty} r^{k}\left(\sum_{|\alpha|=k}\left|a_{\alpha}\right|^{2}\right)^{1 / 2} \leq a_{0}+\frac{2 r}{1-r}\left(1-a_{0}\right) \leq 1
$$

for $0 \leq r \leq \frac{1}{3}$. Now, the proof follows the same lines as the proof of (ii). The proof is complete.

Corollary 1.11. Let

$$
H\left(U_{1}, \ldots, U_{n}\right):=\sum_{k=1}^{\infty} \sum_{|\alpha|=k} \bar{a}_{\alpha} U_{\alpha}^{*}+a_{0} I+\sum_{k=1}^{\infty} \sum_{|\alpha|=k} a_{\alpha} U_{\alpha}, \quad a_{\alpha} \in \mathbb{C},
$$

be a selfadjoint element of $C_{\text {red }}^{*}\left(\mathbb{F}_{n}\right)$ with $\left\|H\left(U_{1}, \ldots, U_{n}\right)\right\| \leq 1$. Then the conclusion of Theorem 1.10 holds.

We remark that a result similar to Corollary 1.11 holds for $C^{*}\left(\mathbb{F}_{n}\right)$.

\section{Operator-Valued Bohr inequalities in SEveral variables}

In this section, we obtain operator-valued multivariable Bohr type inequalities for noncommutative holomorphic functions on the open unit ball of $B(\mathcal{X})^{n}$, the noncommutative disc algebra $\mathcal{A}_{n}$, and the noncommutative analytic Toeplitz algebra $F_{n}^{\infty}$.

We say that a power series

$$
F\left(S_{1}, \ldots, S_{n}\right):=\sum_{k=0}^{\infty} \sum_{|\alpha|=k} S_{\alpha} \otimes A_{(\alpha)}, \quad\left\{A_{(\alpha)}\right\}_{\alpha \in \mathbb{F}_{n}^{+}} \subset B(\mathcal{H}),
$$

generates a (universal) holomorphic function on $\left[B(\mathcal{X})^{n}\right]_{<1}$ with coefficients in $B(\mathcal{H})$ if

$$
F\left(X_{1}, \ldots, X_{n}\right)=\sum_{k=0}^{\infty} \sum_{|\alpha|=k} X_{\alpha} \otimes A_{(\alpha)}
$$

is convergent in the operator norm for any $\left(X_{1}, \ldots, X_{n}\right) \in\left[B(\mathcal{X})^{n}\right]_{<1}$ and any Hilbert space $\mathcal{X}$. We proved in 24 that the following statements are equivalent:

(i) $F\left(S_{1}, \ldots, S_{n}\right)$ generates a holomorphic function on $\left[B(\mathcal{X})^{n}\right]_{<1}$;

(ii) the series $\sum_{k=0}^{\infty}\left\|\sum_{|\alpha|=k} r^{|\alpha|} S_{\alpha} \otimes A_{(\alpha)}\right\|$ is convergent for any $r \in[0,1)$, where $S_{1}, \ldots, S_{n}$ are the left creation operators on the full Fock space $F^{2}\left(H_{n}\right)$;

(iii) $\limsup _{k \rightarrow \infty}\left\|\sum_{|\alpha|=k} A_{(\alpha)}^{*} A_{(\alpha)}\right\|^{1 / 2 k} \leq 1$.

Throughout this section we consider (universal) holomorphic functions on the open unit ball $\left[B(\mathcal{X})^{n}\right]_{<1}$ with coefficients in $B(\mathcal{H})$. We also set $A_{(0)}:=A_{\left(g_{0}\right)}$.

First, we recall a very well-known characterization of contractions on Hilbert spaces. 
Lemma 2.1. A bounded operator $A: \mathcal{K} \rightarrow \mathcal{H}$ is a contraction if and only if

$$
\left(\begin{array}{cc}
I_{\mathcal{H}} & A \\
A^{*} & I_{\mathcal{K}}
\end{array}\right)
$$

is a positive operator acting on the Hilbert space $\mathcal{H} \otimes \mathcal{K}$.

The next positivity result will be useful in what follows.

Lemma 2.2. Let $P, X_{1}, \ldots, X_{m} \in B(\mathcal{H})$ and let $V_{1}, \ldots, V_{m} \in B(\mathcal{K})$ be any isometries with orthogonal ranges. Then

$$
M\left(P, X_{i}\right):=\left(\begin{array}{ccccc}
P & X_{1}^{*} & X_{2}^{*} & \cdots & X_{m}^{*} \\
X_{1} & P & 0 & \cdots & 0 \\
X_{2} & 0 & P & \cdots & 0 \\
\vdots & \vdots & \vdots & \ddots & \vdots \\
X_{m} & 0 & 0 & \cdots & P
\end{array}\right) \geq 0
$$

if and only if

$$
N\left(P, X_{i}\right):=\left(\begin{array}{cc}
I_{\mathcal{K}} \otimes P & \sum_{i=1}^{m} V_{i} \otimes X_{i} \\
\sum_{i=1}^{m} V_{i}^{*} \otimes X_{i}^{*} & I_{\mathcal{K}} \otimes P
\end{array}\right) \geq 0 .
$$

Proof. Notice that $M\left(P, X_{i}\right) \geq 0$ if and only if $P \geq 0$ and

$$
M\left(I_{\mathcal{H}},\left(P+\epsilon I_{\mathcal{H}}\right)^{-1 / 2} X_{i}\left(P+\epsilon I_{\mathcal{H}}\right)^{-1 / 2}\right) \geq 0
$$

for any $\epsilon>0$. Applying Lemma 2.1 to the row operator $A_{\epsilon}:=\left[A_{\epsilon, 1}, \ldots, A_{\epsilon, m}\right]$, where

$$
A_{\epsilon, i}:=\left(P+\epsilon I_{\mathcal{H}}\right)^{-1 / 2} X_{i}^{*}\left(P+\epsilon I_{\mathcal{H}}\right)^{-1 / 2}, \quad i=1, \ldots, m,
$$

one can see that (2.1) holds if and only if $\left\|A_{\epsilon}\right\| \leq 1$ for any $\epsilon>0$. Since $V_{1}, \ldots, V_{m}$ are isometries with orthogonal ranges, we have $V_{i}^{*} V_{j}=\delta_{i j} I_{\mathcal{K}}$ for $i, j=1, \ldots, m$, and

$$
\begin{aligned}
\left\|\sum_{i=1}^{m} V_{i} \otimes A_{\epsilon, i}^{*}\right\|^{2} & =\left\|\sum_{i, j=1}^{m} V_{i}^{*} V_{j} \otimes A_{\epsilon, i} A_{\epsilon, j}^{*}\right\| \\
& =\left\|I_{\mathcal{K}} \otimes \sum_{i=1}^{m} A_{\epsilon, i} A_{\epsilon, i}^{*}\right\|=\left\|A_{\epsilon}\right\|^{2} .
\end{aligned}
$$

Therefore, $\sum_{i=1}^{m} V_{i} \otimes A_{\epsilon, i}^{*}$ is a contraction for any $\epsilon>0$ if and only if $\left\|A_{\epsilon}\right\| \leq 1$ for any $\epsilon>0$. Again applying Lemma 2.1 to the operator $\sum_{i=1}^{m} V_{i} \otimes A_{\epsilon, i}^{*}$, we obtain $N\left(I_{\mathcal{H}}, A_{\epsilon, i}^{*}\right) \geq 0$ for any $\epsilon>0$, which is equivalent to $N\left(P+\epsilon I_{\mathcal{H}}, X_{i}\right) \geq 0$ for any $\epsilon>0$. Taking $\epsilon \rightarrow 0$, we obtain inequality (2.2). The converse follows exactly the same lines. The proof is complete.

We need a few more definitions. Given $\alpha, \beta \in \mathbb{F}_{n}^{+}$, we say that $\alpha>\beta$ if $\alpha=\beta \omega$ for some $\omega \in \mathbb{F}_{n}^{+} \backslash\left\{g_{0}\right\}$. We denote $\omega:=\alpha \backslash \beta$. A kernel $K: \mathbb{F}_{n}^{+} \times \mathbb{F}_{n}^{+} \rightarrow B(\mathcal{H})$ is 
called multi-Toeplitz if $K\left(g_{0}, g_{0}\right)=I_{\mathcal{H}}$ and

$$
K(\alpha, \beta)= \begin{cases}K\left(\alpha \backslash \beta, g_{0}\right) & \text { if } \alpha>\beta \\ I_{\mathcal{H}} & \text { if } \alpha=\beta, \\ K\left(g_{0}, \beta \backslash \alpha\right) & \text { if } \alpha<\beta \\ 0 & \text { otherwise. }\end{cases}
$$

It is said to be positive definite provided that

$$
\sum_{\alpha, \beta \in \mathbb{F}_{n}^{+}}\langle K(\alpha, \beta) h(\beta), h(\alpha)\rangle \geq 0
$$

for all finitely supported functions $h$ from $\mathbb{F}_{n}^{+}$into $\mathcal{H}$.

Using the noncommutative Poisson transforms of [22, one can easily prove the following.

Lemma 2.3. Let

$$
F\left(X_{1}, \ldots, X_{n}\right):=\sum_{k=0}^{\infty} \sum_{|\alpha|=k} X_{\alpha} \otimes A_{(\alpha)}, \quad A_{(\alpha)} \in B(\mathcal{H}),
$$

be a holomorphic function on $\left[B(\mathcal{X})^{n}\right]_{<1}$ with coefficients in $B(\mathcal{H})$. Then the following statements are equivalent:

(i) $\operatorname{Re} F\left(X_{1}, \ldots, X_{n}\right) \leq I$ for any $\left(X_{1}, \ldots, X_{n}\right) \in\left[B(\mathcal{X})^{n}\right]_{<1}$ and any Hilbert space $\mathcal{X}$

(ii) $\operatorname{Re} F\left(r S_{1}, \ldots, r S_{n}\right) \leq I$ for any $0 \leq r<1$.

In what follows we denote by $\left[B_{(\alpha)}:|\alpha|=k\right]$ the row matrix with entries $B_{(\alpha)} \in B(\mathcal{H})$, where $\alpha \in \mathbb{F}_{n}^{+}$and $|\alpha|=k$.

Theorem 2.4. Let

$$
F\left(X_{1}, \ldots, X_{n}\right):=\sum_{k=0}^{\infty} \sum_{|\alpha|=k} X_{\alpha} \otimes A_{(\alpha)}, \quad A_{(\alpha)} \in B(\mathcal{H}),
$$

be a holomorphic function on $\left[B(\mathcal{X})^{n}\right]_{<1}$ with coefficients in $B(\mathcal{H})$ such that $F(0) \geq$ 0 and

$$
\operatorname{Re} F\left(X_{1}, \ldots, X_{n}\right) \leq I \quad \text { for any }\left(X_{1}, \ldots, X_{n}\right) \in\left[B(\mathcal{X})^{n}\right]_{<1} \text {. }
$$

Then

(i) the operator matrix

$$
P_{k}:=\left(\begin{array}{c}
2\left(I_{\mathcal{H}}-A_{(0)}\right) \\
\left.\left[\begin{array}{c}
A_{(\alpha)} \\
\vdots \\
|\alpha|=k
\end{array}\right] \quad\left[\begin{array}{ccc}
2\left(I_{\mathcal{H}}-A_{(0)}\right) & \cdots & |\alpha|=k] \\
\vdots & \ddots & \vdots \\
0 & \cdots & 2\left(I_{\mathcal{H}}-A_{(0)}\right)
\end{array}\right]\right)
\end{array}\right.
$$

is positive for any $k=1,2, \ldots$;

(ii) if $\left\{Y_{(\alpha)}\right\}_{\alpha \in \mathbb{F}_{n}^{+}}$is a sequence of operators in $B(\mathcal{K})$ with

$$
\left\|Y_{(0)}\right\| \leq 1 \text { and } \sum_{k=1}^{\infty}\left\|\sum_{|\alpha|=k} Y_{(\alpha)}^{*} Y_{(\alpha)}\right\|^{1 / 2} \leq \frac{1}{2},
$$


then

$$
\left\|\sum_{k=0}^{\infty} \sum_{|\alpha|=k} S_{\alpha} \otimes A_{(\alpha)} \otimes Y_{(\alpha)}\right\| \leq 1
$$

where the series converges in the norm topology of $B\left(F^{2}\left(H_{n}\right) \otimes \mathcal{H} \otimes \mathcal{K}\right)$.

Proof. Let $R_{1}, \ldots, R_{n}$ be the right creation operators acting on the Fock space $F^{2}\left(H_{n}\right)$. We recall that $R_{i}=U^{*} S_{i} U, \quad i=1, \ldots, n$, where $U$ is the flipping operator. Since $F(0) \geq 0$ and $\operatorname{Re} F\left(r S_{1}, \ldots, r S_{n}\right) \leq I$ for $0 \leq r<1$, we deduce that $A_{(0)} \geq 0$ and

$$
\sum_{k=1}^{\infty} \sum_{|\alpha|=k} r^{|\alpha|} R_{\alpha}^{*} \otimes A_{(\alpha)}^{*}+I \otimes 2 A_{(0)}+\sum_{k=1}^{\infty} \sum_{|\alpha|=k} r^{|\alpha|} R_{\alpha} \otimes A_{(\alpha)} \leq 2 I
$$

for any $0 \leq r<1$, where the series are norm convergent. Hence, we infer that

$$
\sum_{k=1}^{\infty} \sum_{|\alpha|=k} r^{|\alpha|} R_{\alpha}^{*} \otimes C_{(\alpha)}^{*}+I \otimes C_{(0)}+\sum_{k=1}^{\infty} \sum_{|\alpha|=k} r^{|\alpha|} R_{\alpha} \otimes C_{(\alpha)} \geq 0,
$$

where

$$
C_{(0)}:=2\left(I_{\mathcal{H}}-A_{(0)}\right) \text { and } C_{(\alpha)}:=-A_{(\alpha)} \text { if } \alpha \in \mathbb{F}_{n}^{+} \backslash\left\{g_{0}\right\} .
$$

For each $r \in[0,1)$, define the multi-Toeplitz kernel $K_{F, r}: \mathbb{F}_{n}^{+} \times \mathbb{F}_{n}^{+} \rightarrow B(\mathcal{H})$ by

$$
K_{F, r}(\alpha, \beta):= \begin{cases}r^{|\beta \backslash \alpha|} C_{(\widetilde{\beta \backslash \alpha})}^{*} & \text { if } \beta>\alpha, \\ C_{(0)} & \text { if } \alpha=\beta, \\ r^{|\alpha \backslash \beta|} C_{(\widetilde{\alpha \backslash \beta})} & \text { if } \alpha>\beta, \\ 0 & \text { otherwise, }\end{cases}
$$

where $\tilde{\gamma}$ is the reverse of $\gamma \in \mathbb{F}_{n}^{+}$. Note that if $\left\{h_{\beta}\right\}_{|\beta| \leq q} \subset \mathcal{H}$, then

$$
\begin{aligned}
\left\langle\left(\sum_{k=0}^{\infty} \sum_{|\alpha|=k} r^{|\alpha|} R_{\alpha} \otimes C_{(\alpha)}\right)\right. & \left.\left(\sum_{|\beta| \leq q} e_{\beta} \otimes h_{\beta}\right), \sum_{|\gamma| \leq q} e_{\gamma} \otimes h_{\gamma}\right\rangle \\
& =\sum_{k=0}^{\infty} \sum_{|\alpha|=k}\left\langle\sum_{|\beta| \leq q} r^{|\alpha|} R_{\alpha} e_{\beta} \otimes C_{(\alpha)} h_{\beta}, \sum_{|\gamma| \leq q} e_{\gamma} \otimes h_{\gamma}\right\rangle \\
& =\sum_{\alpha \in \mathbb{F}_{n}^{+}|\beta|,|\gamma| \leq q} \sum^{|\alpha|}\left\langle e_{\beta \tilde{\alpha}}, e_{\gamma}\right\rangle\left\langle C_{(\alpha)} h_{\beta}, h_{\gamma}\right\rangle \\
& =\sum_{\gamma \geq \beta ;|\beta|,|\gamma| \leq q} r^{|\gamma \backslash \beta|}\left\langle C_{(\widetilde{\gamma} \widetilde{s})} h_{\beta}, h_{\gamma}\right\rangle \\
& =\sum_{\gamma \geq \beta ;|\beta|,|\gamma| \leq q}\left\langle K_{F, r}(\gamma, \beta) h_{\beta}, h_{\gamma}\right\rangle .
\end{aligned}
$$

Hence, taking into account that $K_{F, r}(\gamma, \beta)=K_{F, r}^{*}(\beta, \gamma)$ and inequality (2.3), we deduce that $\left[K_{F, r}(\alpha, \beta)\right]_{|\alpha|,|\beta| \leq q} \geq 0$ for any $r \in[0,1)$. Taking $r \rightarrow 1$, we obtain $\left[K_{F, 1}(\alpha, \beta)\right]_{|\alpha|,|\beta| \leq q} \geq 0$. According to Theorem 3.1 of [20] and using (2.5), we deduce that there is a completely positive linear map $\mu: C^{*}\left(S_{1}, \ldots, S_{n}\right) \rightarrow B(\mathcal{H})$ such that

$$
\mu\left(S_{\alpha}\right)=K_{F, 1}\left(g_{0}, \alpha\right)=C_{(\widetilde{\alpha})}^{*}, \quad \alpha \in \mathbb{F}_{n}^{+}
$$


Using Stinespring's representation theorem (see [27]), we find a Hilbert space $\mathcal{G} \supseteq$ $\mathcal{H}$, a $*$-representation $\pi: C^{*}\left(S_{1}, \ldots, S_{n}\right) \rightarrow B(\mathcal{G})$, and a bounded operator $X$ : $\mathcal{H} \rightarrow \mathcal{G}$ such that

$$
\mu(f)=X^{*} \pi(f) X, \quad f \in C^{*}\left(S_{1}, \ldots, S_{n}\right) .
$$

Denote $V_{i}:=\pi\left(S_{i}\right), \quad i=1, \ldots, n$, and notice that

$$
X^{*} V_{\alpha} X=\mu\left(S_{\alpha}\right)=C_{(\widetilde{\alpha})}^{*} \quad \text { if } \alpha \in \mathbb{F}_{n}^{+} \backslash\left\{g_{0}\right\},
$$

and $X^{*} X=\mu(I)=C_{(0)}$. On the other hand, if $T: \mathcal{M} \rightarrow \mathcal{G}$ is a contraction, then $\left(\begin{array}{cc}0 & T \\ T^{*} & 0\end{array}\right)$ is a selfadjoint contraction acting on the Hilbert space $\mathcal{G} \oplus \mathcal{M}$. Hence, $\left(\begin{array}{cc}I_{\mathcal{G}} & -T \\ -T^{*} & I_{\mathcal{M}}\end{array}\right)$ is a positive operator. Since $V_{1}, \ldots, V_{n} \in B(\mathcal{G})$ are isometries with orthogonal ranges, the operator $T:=\left[V_{\alpha}:|\alpha|=k\right]$ is a row isometry acting from $\bigoplus_{i=1}^{n^{k}} \mathcal{G}$ to $\mathcal{G}$. Using the above-mentioned result, we deduce that

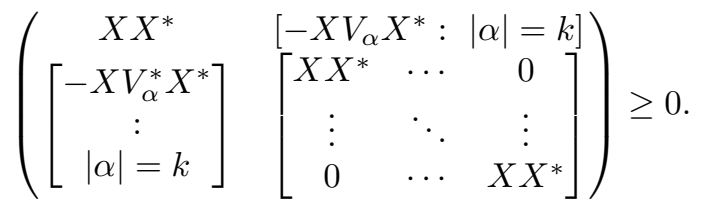

Hence and using relations (2.4) and (2.6), we infer that $P_{k} \geq 0$, which proves part (i) of the theorem. that

To prove (ii), denote $d_{k}:=\left\|\left[Y_{(\alpha)}^{*}:|\alpha|=k\right]\right\|$ for $k=1,2, \ldots$ Lemma 2.1] shows

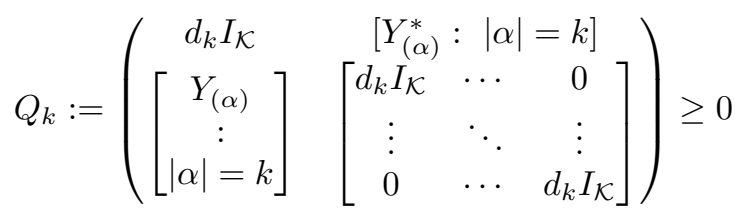

for any $k=1,2, \ldots$. Since $P_{k} \geq 0$ and $Q_{k} \geq 0$, we have $P_{k} \otimes Q_{k} \geq 0$. Compressing the operator matrix $P_{k} \otimes Q_{k}$ to appropriate entries, we deduce that the operator (2.7)

$$
\left(\begin{array}{c}
2 d_{k}\left(I_{\mathcal{H}}-A_{(0)}\right) \otimes I_{\mathcal{K}} \\
\left.\left[\begin{array}{c}
A_{(\alpha)} \otimes Y_{(\alpha)} \\
\vdots \\
|\alpha|=k
\end{array}\right] \quad\left[\begin{array}{cccc}
2 d_{k}\left(I_{\mathcal{H}}-A_{(0)}\right) \otimes Y_{(\alpha)}^{*} & : & |\alpha|=k] \\
\vdots & \cdots & 0 \\
0 & & \cdots & 2 d_{k}\left(I_{\mathcal{H}}-A_{(0)}\right) \otimes I_{\mathcal{K}}
\end{array}\right]\right)
\end{array}\right.
$$

is positive for any $k=1,2, \ldots$. Applying Lemma 2.2 to the operator matrix (2.7), we deduce that

$$
\left(\begin{array}{cc}
I_{F^{2}\left(H_{n}\right)} \otimes 2 d_{k}\left(I_{\mathcal{H}}-A_{(0)}\right) \otimes I_{\mathcal{K}} & \sum_{|\alpha|=k} S_{\alpha} \otimes A_{(\alpha)} \otimes Y_{(\alpha)} \\
\sum_{|\alpha|=k} S_{\alpha}^{*} \otimes A_{(\alpha)}^{*} \otimes Y_{(\alpha)}^{*} & I_{F^{2}\left(H_{n}\right)} \otimes 2 d_{k}\left(I_{\mathcal{H}}-A_{(0)}\right) \otimes I_{\mathcal{K}}
\end{array}\right)
$$

is positive for any $k=1,2, \ldots$, where $S_{1}, \ldots, S_{n}$ are the left creation operators on the Fock space $F^{2}\left(H_{n}\right)$. Hence, using inequality $I-A_{0} \leq I$ and Lemma 2.1, we 
deduce that

$$
\left\|\sum_{|\alpha|=k} S_{\alpha} \otimes A_{(\alpha)} \otimes Y_{(\alpha)}\right\| \leq 2 d_{k}
$$

for $k=1,2, \ldots$. Therefore, the series $\sum_{k=1} \sum_{|\alpha|=k} S_{\alpha} \otimes A_{(\alpha)} \otimes Y_{(\alpha)}$ is convergent in norm. Since $A_{(0)} \geq 0$ and $\left(\begin{array}{cc}I_{\mathcal{K}} & Y_{(0)} \\ Y_{(0)}^{*} & I_{\mathcal{K}}\end{array}\right) \geq 0$, we have

$$
\left(\begin{array}{cc}
I_{F^{2}\left(H_{n}\right)} \otimes A_{(0)} \otimes I_{\mathcal{K}} & I_{F^{2}\left(H_{n}\right)} \otimes A_{(0)} \otimes Y_{(0)} \\
I_{F^{2}\left(H_{n}\right)} \otimes A_{(0)} \otimes Y_{(0)}^{*} & I_{F^{2}\left(H_{n}\right)} \otimes A_{(0)} \otimes I_{\mathcal{K}}
\end{array}\right) \geq 0 .
$$

Taking the sum of the operator matrices given by (2.9) for $k=1,2, \ldots$, and (2.8), and taking into account that $\sum_{k=1}^{\infty} d_{k} \leq \frac{1}{2}$, we deduce that

$$
\left(\begin{array}{cc}
I_{F^{2}\left(H_{n}\right) \otimes \mathcal{H} \otimes \mathcal{K}} & \sum_{k=0}^{\infty} \sum_{|\alpha|=k} S_{\alpha} \otimes A_{(\alpha)} \otimes Y_{(\alpha)} \\
\sum_{k=0}^{\infty} \sum_{|\alpha|=k} S_{\alpha}^{*} \otimes A_{(\alpha)}^{*} \otimes Y_{(\alpha)}^{*} & I_{F^{2}\left(H_{n}\right) \otimes \mathcal{H} \otimes \mathcal{K}}
\end{array}\right)
$$

is a positive operator acting on the Hilbert space $F^{2}\left(H_{n}\right) \otimes \mathcal{H} \otimes \mathcal{K}$. Now, Lemma 2.1 implies

$$
\left\|\sum_{k=0}^{\infty} \sum_{|\alpha|=k} S_{\alpha} \otimes A_{(\alpha)} \otimes Y_{(\alpha)}\right\| \leq 1
$$

The proof is complete.

The next two theorems provide Wiener and Bohr type inequalities for holomorphic functions on $\left[B(\mathcal{X})^{n}\right]_{<1}$ with coefficients in $B(\mathcal{H})$.

Theorem 2.5. Let

$$
F\left(X_{1}, \ldots, X_{n}\right):=\sum_{k=0}^{\infty} \sum_{|\alpha|=k} X_{\alpha} \otimes A_{(\alpha)}, \quad A_{(\alpha)} \in B(\mathcal{H}),
$$

be a holomorphic function on $\left[B(\mathcal{X})^{n}\right]_{<1}$ such that $F(0) \geq 0$ and

$$
\operatorname{Re} F\left(X_{1}, \ldots, X_{n}\right) \leq I \quad \text { for any }\left(X_{1}, \ldots, X_{n}\right) \in\left[B(\mathcal{X})^{n}\right]_{<1} \text {. }
$$

Then

(i) if $Y_{1}, \ldots, Y_{n} \in B(\mathcal{K})$ are bounded operators such that $\left\|\left[Y_{1}^{*}, \ldots, Y_{n}^{*}\right]\right\| \leq \frac{1}{3}$, then

$$
\left\|F\left(S_{1} \otimes Y_{1}, \ldots, S_{n} \otimes Y_{n}\right)\right\| \leq 1
$$

(ii) $\sum_{|\alpha|=k} A_{(\alpha)}^{*} A_{(\alpha)} \leq 4\left(I-A_{(0)}\right)$ for any $k=1,2, \ldots$;

(iii) $\begin{aligned} & \left\|\sum_{|\alpha|=k} A_{(\alpha)}^{*} A_{(\alpha)}\right\|^{1 / 2} \leq 2\left\|I-A_{(0)}\right\| \text { with coefficients in } B(\mathcal{H}) \text { for any } k= \\ & 1,2, \ldots ;\end{aligned}$ 
(iv) for $0 \leq r<1$, we have

$$
\sum_{k=0}^{\infty} r^{k}\left(\sum_{|\alpha|=k} A_{(\alpha)}^{*} A_{(\alpha)}\right)^{1 / 2} \leq M(r) I
$$

where

$$
M(r):= \begin{cases}1+\frac{r^{2}}{(1-r)^{2}} & \text { if } 0 \leq r \leq \frac{1}{2}, \\ \frac{2 r}{1-r} & \text { if } \frac{1}{2}<r<1\end{cases}
$$

(v) if $\left\|A_{(0)}\right\|<1$ and $0 \leq r<1$, then

$$
A_{(0)}+\sum_{k=1}^{\infty} \sum_{|\alpha|=k} r^{k} A_{(\alpha)}^{*}\left(I-A_{(0)}\right)^{-1} A_{(\alpha)} \leq K(r) I,
$$

where

$$
K(r):= \begin{cases}1 & \text { if } 0 \leq r \leq \frac{1}{5} \\ \frac{4 r}{1-r} & \text { if } \frac{1}{5}<r<1\end{cases}
$$

(vi) for $0 \leq r \leq \frac{1}{3}$, we have

$$
\sum_{k=0}^{\infty} r^{k}\left\|\sum_{|\alpha|=k} A_{(\alpha)}^{*} A_{(\alpha)}\right\|^{1 / 2} \leq\left\|A_{(0)}\right\|+\left\|I-A_{(0)}\right\| .
$$

Proof. Define $Y_{(\alpha)}:=Y_{\alpha}, \alpha \in \mathbb{F}_{n}^{+}$, and notice that

$$
\sum_{k=1}^{\infty}\left\|\sum_{|\alpha|=k} Y_{(\alpha)}^{*} Y_{(\alpha)}\right\|^{1 / 2} \leq \sum_{k=1}^{\infty}\left\|\sum_{i=1}^{n} Y_{i}^{*} Y_{i}\right\|^{k / 2}=\sum_{k=1}^{\infty} \frac{1}{3^{k}}=\frac{1}{2}
$$

for $k=1,2, \ldots$. Applying Theorem 2.4 part (ii), we obtain

$$
\left\|\sum_{k=0} \sum_{|\alpha|=k} S_{\alpha} \otimes Y_{\alpha} \otimes A_{(\alpha)}\right\| \leq 1,
$$

which implies (i). To prove (ii) and (iii), note that relations (2.6) and (2.4) imply

$$
\begin{aligned}
\sum_{|\alpha|=k} A_{\widetilde{\alpha}}^{*} A_{\widetilde{\alpha}} & =\sum_{|\alpha|=k} X^{*} V_{\alpha} X X^{*} V_{\alpha}^{*} X \\
& \leq\|X\|^{2} X^{*}\left(\sum_{|\alpha|=k} V_{\alpha} V_{\alpha}^{*}\right) X \leq\|X\|^{2} X^{*} X \\
& =4\left\|I-A_{(0)}\right\|\left(I-A_{(0)}\right) .
\end{aligned}
$$

Therefore, we have

$$
\sum_{|\alpha|=k} A_{\alpha}^{*} A_{\alpha} \leq 4\left\|I-A_{(0)}\right\|\left(I-A_{(0)}\right)
$$


for $k=1,2, \ldots$. According to Theorem 2.4. we have $A_{(0)} \geq 0$ and $P_{k} \geq 0$. This implies $I_{\mathcal{H}}-A_{(0)} \geq 0$ and $\left\|I_{\mathcal{H}}-A_{(0)}\right\| \leq 1$. One can easily see that inequality (2.12) implies (ii) and (iii). Since (ii) implies

$$
\left(\sum_{|\alpha|=k} A_{\alpha}^{*} A_{\alpha}\right)^{1 / 2} \leq 2\left(I-A_{(0)}\right)^{1 / 2}, \quad k=1,2, \ldots,
$$

we deduce that

$$
\begin{aligned}
\sum_{k=0}^{\infty} r^{k}\left(\sum_{|\alpha|=k} A_{(\alpha)}^{*} A_{(\alpha)}\right)^{1 / 2} & \leq A_{(0)}+\frac{2 r}{1-r}\left(I-A_{(0)}\right)^{1 / 2} \\
& \leq \sup _{0 \leq x \leq 1}\left\{x+\frac{2 r}{1-r} \sqrt{1-x}\right\} I=M(r) I,
\end{aligned}
$$

where $M(r)$ is given by (2.10).

Now, assume that $\left\|A_{(0)}\right\|<1$. According to Lemma 2.2. the positivity of the operator $P_{k}$ is equivalent to the positivity of the operator

$$
Q_{k}:=\left(\begin{array}{cc}
I_{F^{2}\left(H_{n}\right)} \otimes 2\left(I_{\mathcal{H}}-A_{(0)}\right) & \sum_{|\alpha|=k} S_{\alpha} \otimes A_{(\alpha)} \\
\sum_{|\alpha|=k} S_{\alpha}^{*} \otimes A_{(\alpha)}^{*} & I_{F^{2}\left(H_{n}\right)} \otimes 2\left(I_{\mathcal{H}}-A_{(0)}\right)
\end{array}\right)
$$

for any $k=1,2, \ldots$ Using Lemma 2.1. we deduce that $Q_{k} \geq 0$ if and only if

$$
\left(\sum_{|\alpha|=k} S_{\alpha}^{*} \otimes A_{(\alpha)}^{*}\right)\left[I \otimes\left(I-A_{(0)}\right)^{-1}\right]\left(\sum_{|\alpha|=k} S_{\alpha} \otimes A_{(\alpha)}\right) \leq I \otimes 4\left(I-A_{(0)}\right)
$$

for any $k=1,2, \ldots$ Hence, and taking into account that $S_{i}^{*} S_{j}=\delta_{i j} I, \quad i, j=$ $1, \ldots, n$, we obtain

$$
\sum_{|\alpha|=k} A_{(\alpha)}^{*}\left(I-A_{(0)}\right)^{-1} A_{(\alpha)} \leq 4\left(I-A_{(0)}\right), \quad k=1,2, \ldots
$$

For $0 \leq r<1$, the latter inequality implies

$$
\begin{aligned}
A_{(0)}+\sum_{k=1}^{\infty} \sum_{|\alpha|=k} r^{k} A_{(\alpha)}^{*}\left(I-A_{(0)}\right)^{-1} A_{(\alpha)} & \leq A_{(0)}+\frac{4 r}{1-r}\left(I-A_{(0)}\right) \\
& \leq \sup _{0 \leq x \leq 1}\left\{x+\frac{4 r}{1-r}(1-x)\right\} I \leq K(r) I,
\end{aligned}
$$

where $K(r)$ is given by (2.11).

To prove (vi), notice that (iii) implies

$$
\begin{aligned}
\sum_{k=0}^{\infty} r^{k}\left\|\sum_{|\alpha|=k} A_{(\alpha)}^{*} A_{(\alpha)}\right\|^{1 / 2} & \leq\left\|A_{(0)}\right\|+2 \frac{r}{1-r}\left\|I-A_{(0)}\right\| \\
& \leq\left\|A_{(0)}\right\|+\left\|I-A_{(0)}\right\|
\end{aligned}
$$

for $0 \leq r \leq \frac{1}{3}$. The proof is complete.

Now we can prove the following operator-valued Bohr inequality for holomorphic functions on $\left[B(\mathcal{X})^{n}\right]_{<1}$. 
Theorem 2.6. Let

$$
F\left(X_{1}, \ldots, X_{n}\right):=\sum_{k=0}^{\infty} \sum_{|\alpha|=k} X_{\alpha} \otimes A_{(\alpha)}, \quad A_{(\alpha)} \in B(\mathcal{H}),
$$

be a holomorphic function on $\left[B(\mathcal{X})^{n}\right]_{<1}$ with coefficients in $B(\mathcal{H})$ such that $F(0) \geq$ 0 and

$$
\operatorname{Re} F\left(X_{1}, \ldots, X_{n}\right) \leq I \quad \text { for any }\left(X_{1}, \ldots, X_{n}\right) \in\left[B(\mathcal{X})^{n}\right]_{<1} .
$$

Then

$$
\sum_{k=0}^{\infty}\left\|\sum_{|\alpha|=k} T_{\alpha} \otimes A_{(\alpha)}\right\| \leq\left\|A_{(0)}\right\|+\left\|I-A_{(0)}\right\|
$$

for any $\left(T_{1}, \ldots, T_{n}\right) \in\left[B(\mathcal{K})^{n}\right]_{1 / 3}$ and any Hilbert space $\mathcal{K}$.

Proof. Let $\left(T_{1}, \ldots, T_{n}\right) \in\left[B(\mathcal{K})^{n}\right]_{r}$ with $0 \leq r \leq \frac{1}{3}$. Notice that $\left[r^{-1} T_{1}, \ldots, r^{-1} T_{n}\right]$ is a row contraction and, according to the noncommutative von Neumann inequality [17, we have

$$
\begin{aligned}
\sum_{k=0}^{\infty}\left\|\sum_{|\alpha|=k} T_{\alpha} \otimes A_{(\alpha)}\right\| & \leq \sum_{k=0}^{\infty} r^{k}\left\|\sum_{|\alpha|=k} S_{\alpha} \otimes A_{(\alpha)}\right\| \\
& =\sum_{k=0}^{\infty} r^{k}\left\|\sum_{|\alpha|=k} A_{(\alpha)}^{*} A_{(\alpha)}\right\|^{1 / 2} .
\end{aligned}
$$

Now using Theorem 2.5 part (vi), we can complete the proof.

For each $\alpha \in \mathbb{F}_{n}^{+}$, define $e_{\alpha}:=e_{i_{1}} \otimes e_{i_{2}} \otimes \cdots \otimes e_{i_{k}}$ and $e_{g_{0}}=1$. It is clear that $\left\{e_{\alpha}: \alpha \in \mathbb{F}_{n}^{+}\right\}$is an orthonormal basis of $F^{2}\left(H_{n}\right)$. We denote by $\mathcal{P}$ the set of all polynomials in $F^{2}\left(H_{n}\right)$, i.e., all the elements of the form

$$
p=\sum_{|\alpha| \leq m} a_{\alpha} e_{\alpha}, \quad a_{\alpha} \in \mathbb{C}, m=0,1,2, \ldots
$$

Remark 2.7. Let

$$
F\left(S_{1}, \ldots, S_{n}\right):=\sum_{k=0}^{\infty} \sum_{|\alpha|=k} S_{\alpha} \otimes A_{(\alpha)}, \quad A_{(\alpha)} \in B(\mathcal{H}),
$$

be a formal power series such that, for each $h \in \mathcal{H}$,

$$
\limsup _{k \rightarrow \infty}\left(\sum_{|\alpha|=k}\left\|A_{\alpha} h\right\|^{2}\right)^{1 / 2 k} \leq 1 .
$$

If $F(0) \geq 0$ and

$$
\operatorname{Re}\left\langle F\left(r S_{1}, \ldots, r S_{n}\right) p, p\right\rangle \leq\|p\|^{2} \quad \text { for any } p \in \mathcal{P} \otimes \mathcal{H} \text { and } 0 \leq r<1,
$$

then the conclusions of Theorem 2.4. Theorem 2.5 and Theorem 2.6 remain true.

We need to recall from [15, [16, 17, 18, and 19] a few facts concerning multianalytic operators on Fock spaces. We say that a bounded linear operator $M$ acting from $F^{2}\left(H_{n}\right) \otimes \mathcal{K}$ to $F^{2}\left(H_{n}\right) \otimes \mathcal{K}^{\prime}$ is multi-analytic if

$$
M\left(S_{i} \otimes I_{\mathcal{K}}\right)=\left(S_{i} \otimes I_{\mathcal{K}^{\prime}}\right) M \quad \text { for any } i=1, \ldots, n .
$$


Notice that $M$ is uniquely determined by the "coefficients" $\theta_{(\alpha)} \in B\left(\mathcal{K}, \mathcal{K}^{\prime}\right)$ given by

$$
\left\langle\theta_{(\tilde{\alpha})} k, k^{\prime}\right\rangle:=\left\langle M(1 \otimes k), e_{\alpha} \otimes k^{\prime}\right\rangle, \quad k \in \mathcal{K}, k^{\prime} \in \mathcal{K}^{\prime}, \alpha \in \mathbb{F}_{n}^{+},
$$

where $\widetilde{\alpha}$ is the reverse of $\alpha$, i.e., $\widetilde{\alpha}=g_{i_{k}} \cdots g_{i_{1}}$ if $\alpha=g_{i_{1}} \cdots g_{i_{k}}$. We denote $\theta_{(0)}:=\theta_{\left(g_{0}\right)}$. Note that

$$
\sum_{\alpha \in \mathbb{F}_{n}^{+}} \theta_{(\alpha)}^{*} \theta_{(\alpha)} \leq\|M\|^{2} I_{\mathcal{K}}
$$

We can associate with $M$ a unique formal Fourier expansion

$$
M \sim \sum_{\alpha \in \mathbb{F}_{n}^{+}} R_{\alpha} \otimes \theta_{(\alpha)},
$$

where $R_{i}:=U^{*} S_{i} U, i=1, \ldots, n$, are the right creation operators on $F^{2}\left(H_{n}\right)$ and $U$ is the (flipping) unitary operator on $F^{2}\left(H_{n}\right)$ mapping $e_{i_{1}} \otimes e_{i_{2}} \otimes \cdots \otimes e_{i_{k}}$ into $e_{i_{k}} \otimes \cdots \otimes e_{i_{2}} \otimes e_{i_{1}}$. Since the operator $M$ acts like its Fourier representation on "polynomials", we will identify them for simplicity. Based on the noncommutative von Neumann inequality ([17, [18]), we proved that

$$
M=\mathrm{SOT}-\lim _{r \rightarrow 1} \sum_{k=0}^{\infty} \sum_{|\alpha|=k} r^{|\alpha|} R_{\alpha} \otimes \theta_{(\alpha)},
$$

where, for each $r \in[0,1)$, the series converges in the uniform norm. Moreover, the set of all multi-analytic operators in $B\left(F^{2}\left(H_{n}\right) \otimes \mathcal{K}, F^{2}\left(H_{n}\right) \otimes \mathcal{K}^{\prime}\right)$ coincides with $R_{n}^{\infty} \bar{\otimes} B\left(\mathcal{K}, \mathcal{K}^{\prime}\right)$, the WOT closed algebra generated by the spatial tensor product, where $R_{n}^{\infty}=U^{*} F_{n}^{\infty} U$.

Now we can deduce the following operator-valued generalization of Bohr's type inequality for the noncommuative analytic Toeplitz algebra $F_{n}^{\infty}$.

Corollary 2.8. If $F\left(S_{1}, \ldots, S_{n}\right):=\sum_{k=0}^{\infty} \sum_{|\alpha|=k} S_{\alpha} \otimes A_{(\alpha)}$ is in $F_{n}^{\infty} \bar{\otimes} B(\mathcal{H})$, then

$$
\sum_{k=0}^{\infty}\left\|\sum_{|\alpha|=k} T_{\alpha} \otimes A_{(\alpha)}\right\| \leq\left\|F\left(S_{1}, \ldots, S_{n}\right)\right\|\left(\left\|A_{(0)}\right\|+\left\|I-A_{(0)}\right\|\right)
$$

for any $\left(T_{1}, \ldots, T_{n}\right) \in\left[B(\mathcal{K})^{n}\right]_{1 / 3}$. Moreover, if $F(0) \geq 0$ and $\operatorname{Re} F\left(S_{1}, \ldots, S_{n}\right) \leq$ $I$, then

$$
\sum_{k=0}^{\infty}\left\|\sum_{|\alpha|=k} T_{\alpha} \otimes A_{(\alpha)}\right\| \leq\left\|A_{(0)}\right\|+\left\|I-A_{(0)}\right\|
$$

for any $\left(T_{1}, \ldots, T_{n}\right) \in\left[B(\mathcal{H})^{n}\right]_{1 / 3}$.

Proof. Without loss of generality, we can assume that $\left\|F\left(S_{1}, \ldots, S_{n}\right)\right\|=1$. Now, one can use the noncommutative Poisson transforms of 22] to show that the hypotheses of Theorem 2.6 are satisfied. Applying the latter theorem, the result follows.

We remark that, in the particular case when $\mathcal{H}=\mathbb{C}$, the first part of Corollary 2.8 was obtained in [12. Its second part provides an operator-valued extension of Corollary 1.6 as well as a new proof.

We can improve some of the inequalities of Theorem 2.5 under the more restrictive conditions that $F\left(S_{1}, \ldots, S_{n}\right) \in F_{n}^{\infty} \bar{\otimes} B(\mathcal{H})$ and $\left\|F\left(S_{1}, \ldots, S_{n}\right)\right\| \leq 1$. 
Theorem 2.9. If

$$
F\left(S_{1}, \ldots, S_{n}\right):=\sum_{k=0}^{\infty} \sum_{|\alpha|=k} S_{\alpha} \otimes A_{(\alpha)} \in F_{n}^{\infty} \bar{\otimes} B(\mathcal{H})
$$

and $\left\|F\left(S_{1}, \ldots, S_{n}\right)\right\| \leq 1$, then

(i) $\left(\sum_{|\alpha|=k} A_{(\alpha)}^{*} A_{(\alpha)}\right)^{1 / 2} \leq\left(I-A_{(0)}^{*} A_{(0)}\right)^{1 / 2} \quad$ for any $k=1,2, \ldots$;

(ii) for $0 \leq r<1$, we have

$$
\sum_{k=0}^{\infty} r^{k}\left(\sum_{|\alpha|=k} A_{(\alpha)}^{*} A_{(\alpha)}\right)^{1 / 2} \leq\left(1+\frac{r^{2}}{(1-r)^{2}}\right)^{1 / 2} I ;
$$

(iii) if $\left\|A_{(0)}\right\|<1$ and $k=1,2, \ldots$, then

$$
\sum_{|\alpha|=k} A_{(\alpha)}^{*}\left(I-A_{(0)} A_{(0)}^{*}\right)^{-1} A_{(\alpha)} \leq I-A_{(0)}^{*} A_{(0)} ;
$$

(iv) if $\left\|A_{(0)}\right\|<1, A_{(0)} \geq 0$, and $0 \leq r \leq 1$, then

$$
A_{(0)}^{2}+\sum_{k=1}^{\infty} \sum_{|\alpha|=k} r^{k} A_{(\alpha)}^{*}\left(I-A_{(0)}^{2}\right)^{-1} A_{(\alpha)} \leq N(r) I,
$$

where

$$
N(r):= \begin{cases}1 & \text { if } 0 \leq r \leq \frac{1}{2} \\ \frac{r}{1-r} & \text { if } \frac{1}{2}<r<1\end{cases}
$$

Proof. Let $\mathcal{M}$ be the subspace of $F^{2}\left(H_{n}\right)$ spanned by the vectors 1 and $e_{\alpha}$, where $\alpha \in \mathbb{F}_{n}^{+}$and $|\alpha|=k$. The operator $P_{\mathcal{M} \otimes \mathcal{H}} F\left(S_{1}, \ldots, S_{n}\right) \mid \mathcal{M} \otimes \mathcal{H}$ is a contraction and its operator matrix with respect to the decomposition $\mathcal{M} \otimes \mathcal{H}=\mathcal{H} \oplus \underset{|\alpha|=k}{\bigoplus}\left(e_{\alpha} \otimes \mathcal{H}\right)$ is

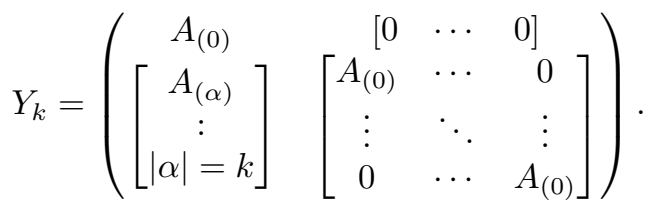

Hence, $\left(\left[\begin{array}{c}A_{(0)} \\ A_{(\alpha)} \\ \vdots \\ |\alpha|=k\end{array}\right]\right)$ is a contraction, which implies

$$
\sum_{|\alpha|=k} A_{(\alpha)}^{*} A_{(\alpha)} \leq I-A_{(0)}^{*} A_{(0)} \quad \text { for } k=1,2, \ldots
$$


The latter inequality implies (i). Using (i), we infer that

$$
\begin{aligned}
\sum_{k=0}^{\infty} r^{k}\left(\sum_{|\alpha|=k} A_{(\alpha)}^{*} A_{(\alpha)}\right)^{1 / 2} & \leq\left(A_{(0)}^{*} A_{(0)}\right)^{1 / 2}+\frac{r}{1-r}\left(1-A_{(0)}^{*} A_{(0)}\right)^{1 / 2} \\
& \leq \sup _{0 \leq x \leq 1}\left\{x+\frac{r}{1-r} \sqrt{1-x^{2}}\right\} I \\
& =\left(1+\frac{r^{2}}{(1-r)^{2}}\right)^{1 / 2} I .
\end{aligned}
$$

Now, we prove part (iii). Taking into account the structure of $2 \times 2$ lower triangular contractions, the operator

$$
\left[\begin{array}{ll}
A & 0 \\
C & D
\end{array}\right]: \mathcal{H} \oplus \mathcal{K} \rightarrow \mathcal{H}^{\prime} \oplus \mathcal{K}^{\prime}
$$

is a contraction if and only if $A$ and $D$ are contractions and

$$
C=\left(I-D D^{*}\right)^{1 / 2} \Gamma\left(I-A^{*} A\right)^{1 / 2},
$$

where $\Gamma: \mathcal{D}_{A} \rightarrow \mathcal{D}_{D^{*}}$ is a contraction, and the subspaces $\mathcal{D}_{A}$ and $\mathcal{D}_{D^{*}}$ are defined by $\mathcal{D}_{A}:=\overline{\left(I-A^{*} A\right)^{1 / 2} \mathcal{H}}$ and $\mathcal{D}_{D^{*}}:=\overline{\left(I-D D^{*}\right)^{1 / 2} \mathcal{K}^{\prime}}$. Hence, if $\|D\|<1$, then we have

$$
C^{*}\left(I-D D^{*}\right)^{-1} C=\left(I-A^{*} A\right)^{1 / 2} \Gamma^{*} \Gamma\left(I-A^{*} A\right)^{1 / 2} \leq I-A^{*} A .
$$

Applying this result to the matrix $Y_{k}$, where $\left\|A_{(0)}\right\|<1$, we obtain

$$
\left[A_{(\alpha)}^{*}:|\alpha|=k\right]\left[\begin{array}{ccc}
\left(I-A_{(0)} A_{(0)}^{*}\right)^{-1} & \cdots & 0 \\
\vdots & \ddots & \vdots \\
0 & \cdots & \left(I-A_{(0)} A_{(0)}^{*}\right)^{-1}
\end{array}\right]\left[\begin{array}{c}
A_{(\alpha)} \\
\vdots \\
|\alpha|=k
\end{array}\right] \leq I-A_{(0)}^{*} A_{(0)}
$$

for $k=1,2, \ldots$, which proves (iii). Now, assume that $A_{(0)} \geq 0$ and $\left\|A_{(0)}\right\|<1$. Using (iii), we deduce that

$$
\begin{aligned}
A_{(0)}^{2}+\sum_{k=1}^{\infty} \sum_{|\alpha|=k} r^{k} A_{(\alpha)}^{*}\left(I-A_{(0)}^{2}\right)^{-1} A_{(\alpha)} & \leq A_{(0)}^{2}+\frac{r}{1-r}\left(I-A_{(0)}^{2}\right) \\
& \leq \sup _{0 \leq x \leq 1}\left\{x+\frac{r}{1-r}(1-x)\right\} I=N(r) I,
\end{aligned}
$$

where $N(r)$ is given by (2.13). The proof is complete.

We recall from [23] the following multivariable operator-valued generalization of the inequalities of Fejér and Egerváry-Százs, to the spatial tensor product

$$
C^{*}\left(S_{1}, \ldots, S_{n}\right) \otimes B(\mathcal{H}) .
$$

Let $m \geq 2$ and let $\left\{A_{(\alpha)}\right\}_{|\alpha| \leq m-1}$ be a sequence of operators in $B(\mathcal{H})$ such that the operator

$$
\sum_{1 \leq k \leq m-1} S_{\alpha}^{*} \otimes A_{(\alpha)}+I \otimes A_{0}+\sum_{1 \leq k \leq m-1} S_{\alpha} \otimes A_{(\alpha)}^{*}
$$

is positive. Then,

$$
w_{e}\left(A_{(\alpha)}:|\alpha|=k\right) \leq w\left(A_{(\alpha)}:|\alpha|=k\right) \leq\left\|A_{0}\right\| \cos \frac{\pi}{\left[\frac{m-1}{k}\right]+2}
$$


for $1 \leq k \leq m-1$, where $[x]$ is the integer part of $x, w\left(X_{1}, \ldots, X_{n}\right)$ is the joint numerical radius of the $n$-tuple $\left(X_{1}, \ldots, X_{n}\right) \in B(\mathcal{X})^{n}$, i.e.,

$$
w\left(X_{1}, \ldots, X_{n}\right):=\omega\left(S_{1} \otimes X_{1}^{*}+\cdots+S_{n} \otimes X_{n}^{*}\right),
$$

and $w_{e}\left(X_{1}, \ldots, X_{n}\right)$ is the euclidean joint numerical radius of the $n$-tuple $\left(X_{1}, \ldots, X_{n}\right)$, i.e.,

$$
w_{e}\left(X_{1}, \ldots, X_{n}\right):=\sup _{\|h\|=1}\left(\sum_{i=1}^{n}\left|\left\langle X_{i} h, h\right\rangle\right|^{2}\right)^{1 / 2} .
$$

We also recall that both the joint numerical radius and the euclidean joint numerical radius are norms equivalent to the operator norm on $B(\mathcal{X})^{n}$. Moreover,

$$
\frac{1}{2}\left\|\left[X_{1}, \ldots, X_{n}\right]\right\| \leq w\left(X_{1}, \ldots, X_{n}\right) \leq\left\|\left[X_{1}, \ldots, X_{n}\right]\right\|
$$

and

$$
\frac{1}{2 \sqrt{n}}\left\|\left[X_{1}, \ldots, X_{n}\right]\right\| \leq w_{e}\left(X_{1}, \ldots, X_{n}\right) \leq w\left(X_{1}, \ldots, X_{n}\right) .
$$

In what follows we obtain an operator-valued Bohr type inequality when the norm of the coefficients is replaced by the joint numerical radius. The result is new even in the single variable case $n=1$.

Theorem 2.10. Let

$$
F\left(S_{1}, \ldots, S_{n}\right):=\sum_{k=0}^{m-1} \sum_{|\alpha|=k} S_{\alpha} \otimes A_{(\alpha)}, \quad A_{(\alpha)} \in B(\mathcal{H}),
$$

be a polynomial such that $F(0) \geq 0$ and $\operatorname{Re} F\left(S_{1}, \ldots, S_{n}\right) \leq I$. Then

$$
\sum_{k=0}^{m-1} r^{k} w\left(A_{(\alpha)}^{*}:|\alpha|=k\right) \leq\left\|A_{(0)}\right\|+\left\|I-A_{(0)}\right\|
$$

for any $r \in\left[0, t_{m}\right]$, where $t_{m} \in(0,1]$ is the solution of the equation

$$
\sum_{k=1}^{m-1} t^{k} \cos \frac{\pi}{\left[\frac{m-1}{k}\right]+2}=\frac{1}{2}
$$

where $[x]$ is the integer part of $x$. Moreover $\left\{t_{m}\right\}$ is a strictly decreasing sequence which converges to $\frac{1}{3}$.

Proof. The conditions $F(0) \geq 0$ and $\operatorname{Re} F\left(S_{1}, \ldots, S_{n}\right) \leq I$ imply

$$
\sum_{1 \leq|\alpha| \leq m-1}-S_{\alpha}^{*} \otimes A_{(\alpha)}^{*}+I \otimes 2\left(I-A_{0}\right)+\sum_{1 \leq|\alpha| \leq m-1}-S_{\alpha} \otimes A_{(\alpha)} \geq 0 .
$$

According to the inequality (2.14), we have

$$
w\left(A_{(\alpha)}^{*}:|\alpha|=k\right) \leq 2\left\|I-A_{0}\right\| \cos \frac{\pi}{\left[\frac{m-1}{k}\right]+2}
$$


for $1 \leq k \leq m-1$. If $0 \leq r \leq t_{m}$, then we have

$$
\begin{aligned}
\sum_{k=0}^{m-1} r^{k} w\left(A_{(\alpha)}^{*}:|\alpha|=k\right) & \leq\left\|A_{0}\right\|+2\left\|I-A_{0}\right\| \sum_{k=1}^{m-1} r^{k} \cos \frac{\pi}{\left[\frac{m-1}{k}\right]+2} \\
& \leq\left\|A_{0}\right\|+2\left\|I-A_{0}\right\| \sum_{k=1}^{m-1} t_{m}^{k} \cos \frac{\pi}{\left[\frac{m-1}{k}\right]+2} \\
& \leq\left\|A_{(0)}\right\|+\left\|I-A_{(0)}\right\| .
\end{aligned}
$$

The last part of the theorem was proved in Theorem 1.1. The proof is complete.

\section{Operator-VAlued BoHR INEQUALITIES FOR HARMONIC FUNCTIONS}

We say that $G$ is a selfadjoint harmonic function on $\left[B(\mathcal{X})^{n}\right]_{<1}$ with coefficients in $B(\mathcal{H})$ if there exists a (universal) holomorphic function $F:\left[B(\mathcal{X})^{n}\right]_{<1} \rightarrow B(\mathcal{X} \otimes \mathcal{H})$ such that

$$
G\left(X_{1}, \ldots, X_{n}\right)=\operatorname{Re} F\left(X_{1}, \ldots, X_{n}\right) \quad \text { for any }\left(X_{1}, \ldots, X_{n}\right) \in\left[B(\mathcal{X})^{n}\right]_{<1} .
$$

Let $\mathcal{A}:=\left\{A_{(\alpha)}\right\}_{\alpha \in \mathbb{F}_{n}^{+}}$be a sequence of operators in $B(\mathcal{H})$ such that $A_{(0)}=A_{(0)}^{*}$ and

$$
F\left(X_{1}, \ldots, X_{n}\right)=\frac{1}{2} I \otimes A_{(0)}+\sum_{k=1}^{\infty} \sum_{|\alpha|=k} X_{\alpha} \otimes A_{(\alpha)}
$$

is a holomorphic function on $\left[B(\mathcal{X})^{n}\right]_{<1}$. Define $H_{\mathcal{A}}:\left[B(\mathcal{X})^{n}\right]_{<1} \rightarrow B(\mathcal{H} \otimes \mathcal{X})$ by setting $H_{\mathcal{A}}\left(X_{1}, \ldots, X_{n}\right)=\operatorname{Re} F\left(X_{1}, \ldots, X_{n}\right)$ for any $\left(X_{1}, \ldots, X_{n}\right) \in\left[B(\mathcal{X})^{n}\right]_{<1}$.

We remark that, in the particular case when $\mathcal{A}:=\left\{a_{\alpha}\right\}_{\alpha \in \mathbb{F}_{n}^{+}} \subset \mathbb{C}$ are the coefficients of an element in the noncommutative analytic Toeplitz algebra $F_{n}^{\infty}$ of the form

$$
F\left(S_{1}, \ldots, S_{n}\right)=\frac{a_{0}}{2} I+\sum_{k=1}^{\infty} \sum_{|\alpha|=k} a_{\alpha} S_{\alpha}, \quad a_{0}=\bar{a}_{0},
$$

then the operator

$$
H_{\mathcal{A}}\left(S_{1}, \ldots, S_{n}\right):=\frac{1}{2}\left[F\left(S_{1}, \ldots, S_{n}\right)^{*}+F\left(S_{1}, \ldots, S_{n}\right)\right]
$$

can be seen as a noncommutative analogue of the boundary function of a (realvalued) bounded harmonic function in the unit disc, while $H_{\mathcal{A}}\left(X_{1}, \ldots, X_{n}\right)$ can be seen as the noncommutative Poisson transform of $H_{\mathcal{A}}\left(S_{1}, \ldots, S_{n}\right)$ at the point $\left(X_{1}, \ldots, X_{n}\right) \in\left[B(\mathcal{X})^{n}\right]_{<1}$.

We remark that, using the noncommutative Poisson transforms of 22], one can easily show that

$$
H_{\mathcal{A}}\left(X_{1}, \ldots, X_{n}\right) \leq H_{\mathcal{B}}\left(X_{1}, \ldots, X_{n}\right) \quad \text { for any }\left(X_{1}, \ldots, X_{n}\right) \in\left[B(\mathcal{X})^{n}\right]_{<1}
$$

if and only if

$$
H_{\mathcal{A}}\left(r S_{1}, \ldots, r S_{n}\right) \leq H_{\mathcal{B}}\left(r S_{1}, \ldots, r S_{n}\right) \text { for any } r \in[0,1) .
$$

The first result of this section provides Wiener and Bohr type inequalities for the coefficients of two harmonic functions on $\left[B(\mathcal{X})^{n}\right]_{<1}$ satisfying the inequality $H_{\mathcal{A}}\left(X_{1}, \ldots, X_{n}\right) \leq H_{\mathcal{B}}\left(X_{1}, \ldots, X_{n}\right)$. 
Theorem 3.1. Let $\mathcal{A}:=\left\{A_{(\alpha)}\right\}_{\alpha \in \mathbb{F}_{n}^{+}}$and $\mathcal{B}:=\left\{B_{(\alpha)}\right\}_{\alpha \in \mathbb{F}_{n}^{+}}$be sequences of operators in $B(\mathcal{H})$ such that $H_{\mathcal{A}}$ and $H_{\mathcal{B}}$ are noncommutative harmonic functions on $\left[B(\mathcal{X})^{n}\right]_{<1}$ and

$$
H_{\mathcal{A}}\left(X_{1}, \ldots, X_{n}\right) \leq H_{\mathcal{B}}\left(X_{1}, \ldots, X_{n}\right) \quad \text { for any }\left(X_{1}, \ldots, X_{n}\right) \in\left[B(\mathcal{X})^{n}\right]_{<1} .
$$

Then

$$
\sum_{|\alpha|=k}\left(B_{(\alpha)}-A_{(\alpha)}\right)^{*}\left(B_{(\alpha)}-A_{(\alpha)}\right) \leq\left\|B_{(0)}-A_{(0)}\right\|\left(B_{(0)}-A_{(0)}\right)
$$

for $k=1,2, \ldots$, and

$$
\sum_{k=1}^{\infty} r^{k}\left\|\sum_{|\alpha|=k} A_{(\alpha)}^{*} A_{(\alpha)}\right\|^{1 / 2} \leq \frac{1}{2}\left\|B_{(0)}-A_{(0)}\right\|+\sum_{k=1}^{\infty} r^{k}\left\|\sum_{|\alpha|=k} B_{(\alpha)}^{*} B_{(\alpha)}\right\|^{1 / 2}
$$

for any $r \in\left[0, \frac{1}{3}\right]$.

Proof. Notice that the inequality (3.1) implies

$$
\sum_{k=1}^{\infty} \sum_{|\alpha|=k} r^{|\alpha|} S_{\alpha}^{*} \otimes C_{(\alpha)}^{*}+I \otimes C_{(0)}+\sum_{k=1}^{\infty} \sum_{|\alpha|=k} r^{|\alpha|} S_{\alpha} \otimes C_{(\alpha)} \geq 0, \quad r \in[0,1)
$$

where $C_{(\alpha)}:=B_{(\alpha)}-A_{(\alpha)}$ if $\alpha \in \mathbb{F}_{n}^{+} \backslash\left\{g_{0}\right\}$ and $C_{(0)}:=B_{(0)}-A_{(0)}$. First we consider the case when $C_{(0)} \neq 0$. According to (3.3), we have $C_{(0)} \geq 0$. As in the proof of Theorem 2.4 part (i), we deduce that there is a completely positive linear map $\mu: C^{*}\left(S_{1}, \ldots, S_{n}\right) \rightarrow B(\mathcal{H})$ such that

$$
\mu\left(S_{\alpha}\right)=C_{(\widetilde{\alpha})}^{*}, \quad \alpha \in \mathbb{F}_{n}^{+} .
$$

Using Stinespring's representation theorem, we find a Hilbert space $\mathcal{G} \supseteq \mathcal{H}$, a $*$ representation $\pi: C^{*}\left(S_{1}, \ldots, S_{n}\right) \rightarrow B(\mathcal{G})$, and a bounded operator $X: \mathcal{H} \rightarrow \mathcal{G}$ such that

$$
\mu(f)=X^{*} \pi(f) X, \quad f \in C^{*}\left(S_{1}, \ldots, S_{n}\right) .
$$

Denote $V_{i}:=\pi\left(S_{i}\right), \quad i=1, \ldots, n$, and notice that

$$
X^{*} V_{\alpha} X=\mu\left(S_{\alpha}\right)=C_{(\widetilde{\alpha})}^{*} \quad \text { if } \alpha \in \mathbb{F}_{n}^{+} \backslash\left\{g_{0}\right\},
$$

and $X^{*} X=\mu(I)=C_{(0)}$. Hence, we infer that

$$
\begin{aligned}
\sum_{|\alpha|=k} C_{\tilde{\alpha}}^{*} C_{\tilde{\alpha}} & =\sum_{|\alpha|=k} X^{*} V_{\alpha} X X^{*} V_{\alpha}^{*} X \\
& \leq\|X\|^{2} X^{*}\left(\sum_{|\alpha|=k} V_{\alpha} V_{\alpha}^{*}\right) X \\
& \leq\|X\|^{2} X^{*} X=\left\|C_{(0)}\right\| C_{(0)} .
\end{aligned}
$$

Therefore, the inequality (3.2) holds. It is clear that the latter inequality implies

$$
\left\|\sum_{|\alpha|=k}\left(B_{(\alpha)}-A_{(\alpha)}\right)^{*}\left(B_{(\alpha)}-A_{(\alpha)}\right)\right\|^{1 / 2} \leq\left\|B_{(0)}-A_{(0)}\right\|
$$


for $k=1,2, \ldots$. Using this inequality, we deduce that

$$
\begin{aligned}
\sum_{k=1}^{\infty} r^{k}\left\|\left[A_{(\alpha)}:|\alpha|=k\right]\right\| & -\sum_{k=1}^{\infty} r^{k}\left\|\left[B_{(\alpha)}:|\alpha|=k\right]\right\| \\
& \leq \sum_{k=1}^{\infty} r^{k}\left\|\left[A_{(\alpha)}-B_{(\alpha)}:|\alpha|=k\right]\right\| \\
& \leq \sum_{k=1}^{\infty} r^{k}\left\|B_{(0)}-A_{(0)}\right\| \\
& \leq\left\|B_{(0)}-A_{(0)}\right\| \frac{r}{1-r} \\
& \leq \frac{1}{2}\left\|B_{(0)}-A_{(0)}\right\|
\end{aligned}
$$

for any $r \in\left[0, \frac{1}{3}\right]$. Therefore, the second inequality of the theorem is proved.

Now, notice that if $A_{(0)}=B_{(0)}$, then adding $\epsilon I, \epsilon>0$, to inequality (3.3) and applying the first part of the proof, we deduce that

$$
\sum_{|\alpha|=k}\left(B_{(\alpha)}-A_{(\alpha)}\right)^{*}\left(B_{(\alpha)}-A_{(\alpha)}\right) \leq \epsilon^{2} I
$$

for any $\epsilon>0$. This implies $A_{(\alpha)}=B_{(\alpha)}$, for any $\alpha \in \mathbb{F}_{n}^{+}$. The proof is complete.

We remark that Theorem 3.1 remains true if the conditions on the coefficients $\mathcal{A}:=\left\{A_{(\alpha)}\right\}_{\alpha \in \mathbb{F}_{n}^{+}}$and $\mathcal{B}:=\left\{B_{(\alpha)}\right\}_{\alpha \in \mathbb{F}_{n}^{+}}$are replaced by the following weaker conditions:

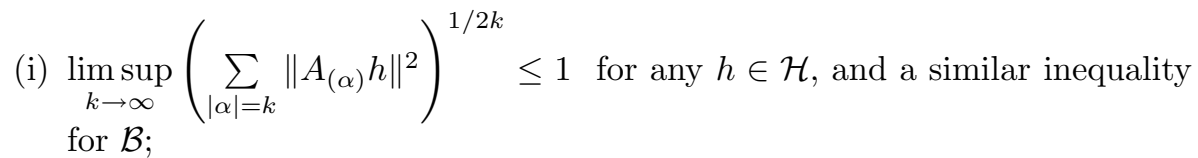

(ii) $\left\langle H_{\mathcal{A}}\left(r S_{1}, \ldots, r S_{n}\right) p, p\right\rangle \leq\left\langle H_{\mathcal{B}}\left(r S_{1}, \ldots, r S_{n}\right) p, p\right\rangle$ for any $r \in[0,1)$ and $p \in$ $\mathcal{P} \otimes \mathcal{H}$.

Now, we can prove the following result for selfadjoint harmonic polynomials.

Theorem 3.2. Let $m=2,3, \ldots$, and let $\mathcal{A}:=\left\{A_{\alpha}\right\}_{|\alpha| \leq m-1}$ and $\mathcal{B}:=\left\{B_{\alpha}\right\}_{|\alpha| \leq m-1}$ be sequences of operators such that $A_{(0)}=A_{(0)}^{*}, B_{(0)}=B_{(0)}^{*}$, and

$$
H_{\mathcal{A}}\left(S_{1}, \ldots, S_{n}\right) \leq H_{\mathcal{B}}\left(S_{1}, \ldots, S_{n}\right) .
$$

Then

$$
w\left(B_{(\alpha)}^{*}-A_{(\alpha)}^{*}:|\alpha|=k\right) \leq\left\|B_{(0)}-A_{(0)}\right\| \cos \frac{\pi}{\left[\frac{m-1}{k}\right]+2}
$$

for $1 \leq k \leq m-1$, where $[x]$ is the integer part of $x$, and

$$
\sum_{k=1}^{m-1} r^{k} w\left(A_{(\alpha)}^{*}:|\alpha|=k\right) \leq \frac{1}{2}\left\|B_{(0)}-A_{(0)}\right\|+\sum_{k=1}^{m-1} r^{k} w\left(B_{(\alpha)}^{*}:|\alpha|=k\right)
$$

for any $r \in\left[0, t_{m}\right]$, where $t_{m}$ is the solution of equation (0.1). 
Proof. Since

$$
\begin{aligned}
\sum_{k=1}^{m-1} \sum_{|\alpha|=k} r^{|\alpha|} S_{\alpha}^{*} \otimes\left(B_{(\alpha)}^{*}-A_{(\alpha)}^{*}\right)+I \otimes & \left(B_{(0)}-A_{(0)}\right) \\
& +\sum_{k=1}^{m-1} \sum_{|\alpha|=k} r^{|\alpha|} S_{\alpha} \otimes\left(B_{(\alpha)}-A_{(\alpha)}\right) \geq 0
\end{aligned}
$$

for $r \in[0,1)$, inequality (2.14) implies (3.6). Since the joint numerical radius is a norm and using the inequality (3.6), we have

$$
\begin{aligned}
\sum_{k=1}^{m-1} r^{k} w\left(A_{(\alpha)}^{*}:|\alpha|=k\right) & -\sum_{k=1}^{m-1} r^{k} w\left(B_{(\alpha)}^{*}:|\alpha|=k\right) \\
& \leq \sum_{k=1}^{m-1} r^{k} w\left(B_{(\alpha)}^{*}-A_{(\alpha)}^{*}:|\alpha|=k\right) \\
& \leq\left\|B_{(0)}-A_{(0)}\right\| \sum_{k=1}^{m-1} r^{k} \cos \frac{\pi}{\left[\frac{m-1}{k}\right]+2} \\
& \leq\left\|B_{(0)}-A_{(0)}\right\| \sum_{k=1}^{m-1} t_{m}^{k} \cos \frac{\pi}{\left[\frac{m-1}{k}\right]+2} \\
& \leq \frac{1}{2}\left\|B_{(0)}-A_{(0)}\right\|
\end{aligned}
$$

for any $r \in\left[0, t_{m}\right]$. This completes the proof.

We remark that one can obtain versions of Theorem 3.1 and Theorem 3.2 for the spatial tensor products $C_{\text {red }}^{*}\left(\mathbb{F}_{n}\right) \otimes B(\mathcal{H})$ and $C^{*}\left(\mathbb{F}_{n}\right) \otimes B(\mathcal{H})$. In this way we can provide operator-valued generalizations of Corollary 1.9 and Corollary 1.11.

Given $m=2,3, \ldots, \infty$, and a sequence $\mathcal{A}:=\left\{a_{\alpha}\right\}_{|\alpha| \leq m-1}$ of complex numbers with $a_{0}=\bar{a}_{0}$, define

$$
H_{\mathcal{A}}\left(S_{1}, \ldots, S_{n}\right):=\sum_{1 \leq|\alpha| \leq m-1} \bar{a}_{\alpha} S_{\alpha}^{*}+a_{0} I+\sum_{1 \leq|\alpha| \leq m-1} a_{\alpha} S_{\alpha} .
$$

When $m=\infty$, we assume that the series $\sum_{k=1}^{\infty}\left\|\sum_{|\alpha|=k} r^{|\alpha|} a_{\alpha} S_{\alpha}\right\|$ is convergent for any $r \in[0,1)$. The following result is a scalar version of Theorem 3.1 and Theorem 3.2 ,

Corollary 3.3. Let $m=2,3, \ldots, \infty$ and let

$$
\mathcal{A}:=\left\{a_{\alpha}\right\}_{|\alpha| \leq m-1} \quad \text { and } \quad \mathcal{B}:=\left\{b_{\alpha}\right\}_{|\alpha| \leq m-1}
$$

be sequences of complex numbers such that $a_{0}=\bar{a}_{0}, b_{0}=\bar{b}_{0}$, and

$$
H_{\mathcal{A}}\left(S_{1}, \ldots, S_{n}\right) \leq H_{\mathcal{B}}\left(S_{1}, \ldots, S_{n}\right) \quad \text { if } m<\infty
$$

and, if $m=\infty$,

$$
H_{\mathcal{A}}\left(r S_{1}, \ldots, r S_{n}\right) \leq H_{\mathcal{B}}\left(r S_{1}, \ldots, r S_{n}\right) \quad \text { for any } r \in[0,1) .
$$


Then

$$
\left(\sum_{|\alpha|=k}\left|b_{\alpha}-a_{\alpha}\right|^{2}\right)^{1 / 2} \leq\left(b_{0}-a_{0}\right) \cos \frac{\pi}{\left[\frac{m-1}{k}\right]+2}
$$

for $1 \leq k \leq m-1$, where $[x]$ is the integer part of $x$, and

$$
\frac{a_{0}}{2}+\sum_{k=1}^{m-1} r^{k}\left(\sum_{\mid \alpha=k}\left|a_{\alpha}\right|^{2}\right)^{1 / 2} \leq \frac{b_{0}}{2}+\sum_{k=1}^{m-1} r^{k}\left(\sum_{|\alpha|=k}\left|b_{\alpha}\right|^{2}\right)^{1 / 2}
$$

for any $r \in\left[0, t_{m}\right]$, where $t_{m}$ is the solution of the equation (0.1) if $m<\infty$ and $t_{\infty}:=\frac{1}{3}$.

Now, we can deduce Bohr inequalities for two real-valued functions $f, g \in L^{1}(\mathbb{T})$ satisfying the inequality $f \leq g$.

Corollary 3.4. Let $f\left(e^{i t}\right)=\sum_{k=-m+1}^{m-1} a_{k} e^{i k t}$ and $g\left(e^{i t}\right)=\sum_{k=-m+1}^{m-1} a_{k} e^{i k t}$ be realvalued functions in $L^{1}(\mathbb{T})$, where $m=2,3, \ldots, \infty$. If $f \leq g$, then

$$
\frac{a_{0}}{2}+\sum_{k=1}^{m-1} r^{k}\left|a_{k}\right| \leq \frac{b_{0}}{2}+\sum_{k=1}^{m-1} r^{k}\left|b_{k}\right|
$$

for any $r \in\left[0, t_{m}\right]$, where $t_{m}$ is the solution of the equation (0.1) if $m<\infty$ and $t_{\infty}:=\frac{1}{3}$.

Proof. Taking the harmonic extension of $f$ and $g$ in the unit disc, we obtain $f\left(r e^{i t}\right) \leq g\left(r e^{i t}\right)$ for $0 \leq r<1$ and $t \in[-\pi, \pi]$. Notice that, for each $m=$ $2,3, \ldots, \infty$,

$$
H_{\mathcal{A}}(r S):=\sum_{k=1}^{m-1} r^{k} \bar{a}_{k} S^{* k}+a_{0} I+\sum_{k=1}^{m-1} r^{k} a_{k} S^{k}, \quad r \in[0,1),
$$

is in the $C^{*}$-algebra generated by the unilateral shift $S$, acting on $H^{2}(\mathbb{T})$. Similarly, we can define $H_{\mathcal{B}}(r S)$, where $\mathcal{B}:=\left\{b_{k}\right\}_{k=0}^{m-1}$. Now, notice that, for any $h\left(e^{i t}\right) \in$ $H^{2}(\mathbb{T})$, we have

$$
\begin{aligned}
\left\langle\left(H_{\mathcal{B}}(r S)-H_{\mathcal{A}}(r S)\right) h\left(e^{i t}\right), h\left(e^{i t}\right)\right\rangle_{H^{2}(\mathbb{T})} & =\left\langle\left(g\left(r e^{i t}\right)-f\left(r e^{i t}\right)\right) h\left(e^{i t}\right), h\left(e^{i t}\right)\right\rangle_{H^{2}(\mathbb{T})} \\
& =\frac{1}{2 \pi} \int_{-\pi}^{\pi}\left(g\left(r e^{i t}\right)-f\left(r e^{i t}\right)\left|h\left(e^{i t}\right)\right|^{2} d t \geq 0 .\right.
\end{aligned}
$$

Therefore, $H_{\mathcal{B}}(r S) \geq H_{\mathcal{A}}(r S)$ for $0 \leq r<1$. Now applying Theorem 3.1 (when $m=\infty)$ and Theorem 3.2 (when $m=2,3, \ldots$ ), the result follows.

Corollary 3.5. Let $f:=\sum_{k=-m+1}^{m-1} a_{k} e^{i k t}$ and $g:=\sum_{k=-m+1}^{m-1} b_{k} e^{i k t}$ be real-valued functions in $L^{1}(\mathbb{T})$, where $m=2,3, \ldots, \infty$. If there exists $r \in\left[0, t_{m}\right]$, where $t_{m}$ is the solution of the equation (0.1) if $m<\infty$ and $t_{\infty}:=\frac{1}{3}$, such that

$$
\frac{a_{0}}{2}+\sum_{k=1}^{m-1} r^{k}\left|a_{k}\right|>\frac{b_{0}}{2}+\sum_{k=1}^{m-1} r^{k}\left|b_{k}\right|
$$

then $f>g$ on a set of positive measure. 
We mention that, when $m=\infty$, the last two corollaries were obtained by Paulsen and Singh [13.

\section{Bohr inequalities for ANALYTiC FUnCtions on the UNit BALL OF $\mathbb{C}^{n}$}

A multivariable commutative analogue of the Hardy space $H^{\infty}(\mathbb{D})$ is the algebra $W_{n}^{\infty}:=\left.P_{F_{s}^{2}\left(H_{n}\right)} F_{n}^{\infty}\right|_{F_{s}^{2}\left(H_{n}\right)}$, the compression of $F_{n}^{\infty}$ to the symmetric Fock space $F_{s}^{2}\left(H_{n}\right) \subset F^{2}\left(H_{n}\right)$. We proved in [2] that $W_{n}^{\infty}$ is the $w^{*}$-closed algebra generated by the creation operators $B_{i}:=\left.P_{F_{s}^{2}\left(H_{n}\right)} S_{i}\right|_{F_{s}^{2}\left(H_{n}\right)}, i=1, \ldots, n$, and the identity. Arveson [3] showed that the algebra $W_{n}^{\infty}$ can be seen as the multiplier algebra of the reproducing kernel Hilbert space with reproducing kernel $K_{n}: \mathbb{B}_{n} \times \mathbb{B}_{n} \rightarrow \mathbb{C}$ defined by

$$
K_{n}(z, w):=\frac{1}{1-\langle z, w\rangle_{\mathbb{C}^{n}}}, \quad z, w \in \mathbb{B}_{n},
$$

where $\mathbb{B}_{n}$ is the open unit ball of $\mathbb{C}^{n}$.

Let $\mathbf{p}:=\left(p_{1}, \ldots, p_{n}\right)$ be a multi-index in $\mathbb{Z}_{+}^{n}$. We denote $|\mathbf{p}|:=p_{1}+\cdots+p_{n}$ and $\mathbf{p} !:=p_{1} ! \cdots p_{n}$ !. If $\lambda:=\left(\lambda_{1}, \ldots, \lambda_{n}\right)$, then we set $\lambda^{\mathbf{p}}:=\lambda_{1}^{p_{1}} \cdots \lambda_{n}^{p_{n}}$ and

$$
\left(\lambda^{\mathbf{p}}\right)_{\text {sym }}\left(S_{1}, \ldots, S_{n}\right):=\frac{\mathbf{p} !}{|\mathbf{p}| !} \sum_{\alpha \in \Lambda_{\mathbf{p}}} S_{\alpha},
$$

where

$$
\Lambda_{\mathbf{p}}:=\left\{\alpha \in \mathbb{F}_{n}^{+}: \lambda_{\alpha}=\lambda^{\mathbf{p}} \text { for any } \lambda \in \mathbb{B}_{n}\right\}
$$

and $S_{1}, \ldots, S_{n}$ are the left creation operators on the Fock space $F^{2}\left(H_{n}\right)$. Notice that $\operatorname{card} \Lambda_{\mathbf{p}}=\frac{|\mathbf{p}| !}{\mathbf{p} !}$. If $p(\lambda):=\sum \lambda^{\mathbf{p}} A_{(\mathbf{p})}, A_{(\mathbf{p})} \in B(\mathcal{H})$, is an operator-valued polynomial on $\mathbb{B}_{n}$, the open unit ball of $\mathbb{C}^{n}$, then the symmetrized functional calculus $p_{\text {sym }}\left(S_{1}, \ldots, S_{n}\right) \in F_{n}^{\infty} \bar{\otimes} B(\mathcal{H})$ is defined by

$$
p_{\text {sym }}\left(S_{1}, \ldots, S_{n}\right):=\sum\left[\left(\lambda^{\mathbf{p}}\right)_{\text {sym }}\left(S_{1}, \ldots, S_{n}\right)\right] \otimes A_{(\mathbf{p})} .
$$

In this section we obtain Wiener and Bohr inequalities for the algebra $W_{n}^{\infty}$ and a class of operator-valued analytic functions on the open unit ball of $\mathbb{C}^{n}$.

Theorem 4.1. Let $f\left(\lambda_{1}, \ldots, \lambda_{n}\right):=\sum_{\mathbf{p} \in \mathbb{Z}_{+}^{\mathbf{n}}} \lambda^{\mathbf{p}} A_{(\mathbf{p})}, A_{(\mathbf{p})} \in B(\mathcal{H})$, be an operatorvalued analytic function on $\mathbb{B}_{n}$ such that

$$
\limsup _{k \rightarrow \infty}\left\|\sum_{\mathbf{p} \in \mathbb{Z}_{+}^{n},|\mathbf{p}|=k} \frac{|\mathbf{p}| !}{\mathbf{p} !} A_{(\mathbf{p})}^{*} A_{(\mathbf{p})}\right\|^{1 / 2 k} \leq 1,
$$

$f(0) \geq 0$, and

$$
\operatorname{Re} f_{\mathrm{sym}}\left(r S_{1}, \ldots, r S_{n}\right) \leq I \quad \text { for } 0 \leq r<1 .
$$

Then the following statements hold:

(i)

$$
\begin{aligned}
& \text { (i) }\left\|\sum_{\mathbf{p} \in \mathbb{Z}_{+}^{n},|\mathbf{p}|=k} \frac{\mathbf{p} !}{|\mathbf{p}| !} A_{(\mathbf{p})}^{*} A_{(\mathbf{p})}\right\|^{1 / 2} \leq 2\left\|I-A_{(0)}\right\| \text { for } k=1,2, \ldots \\
& \text { (ii) } \sum_{k=0}^{\infty} r^{k}\left\|\sum_{\mathbf{p} \in \mathbb{Z}_{+}^{n},|\mathbf{p}|=k} \frac{\mathbf{p} !}{|\mathbf{p}| !} A_{(\mathbf{p})}^{*} A_{(\mathbf{p})}\right\|^{1 / 2} \leq\left\|A_{(0)}\right\|+\left\|I-A_{(0)}\right\| \quad \text { for } 0 \leq r \leq \frac{1}{3} .
\end{aligned}
$$


(iii) If $\left\{Y_{(\mathbf{p})}\right\}_{\mathbf{p} \in \mathbb{Z}_{+}^{n}}$ is a sequence of operators in $B(\mathcal{K})$ such that

$$
\left\|Y_{(0)}\right\| \leq 1 \text { and } \sum_{k=0}^{\infty}\left\|\sum_{\mathbf{p} \in \mathbb{Z}_{+}^{n},|\mathbf{p}|=k} \frac{|\mathbf{p}| !}{\mathbf{p} !} Y_{(\mathbf{p})}^{*} Y_{(\mathbf{p})}\right\|^{1 / 2} \leq \frac{1}{2},
$$

then

$$
\left\|\sum_{k=0}^{\infty}\left(\sum_{\mathbf{p} \in \mathbb{Z}_{+}^{n},|\mathbf{p}|=k} B^{\mathbf{p}} \otimes A_{(\mathbf{p})} \otimes Y_{(\mathbf{p})}\right)\right\| \leq 1,
$$

where $B_{1}, \ldots, B_{n}$ are the creation operators on the symmetric Fock space. In particular,

$$
\left\|\sum_{k=0}^{\infty}\left(\sum_{\mathbf{p} \in \mathbb{Z}_{+}^{n},|\mathbf{p}|=k} \lambda^{\mathbf{p}} A_{(\mathbf{p})} \otimes Y_{(\mathbf{p})}\right)\right\| \leq 1 \text { for any } \lambda \in \mathbb{B}_{n} .
$$

(iv) If $Y_{1}, \ldots, Y_{n} \in B(\mathcal{K})$ are commuting and $\left\|\left[Y_{1}^{*}, \ldots, Y_{n}^{*}\right]\right\| \leq \frac{1}{3}$, then

$$
\left\|\sum_{k=0}^{\infty}\left(\sum_{\mathbf{p} \in \mathbb{Z}_{+}^{n},|\mathbf{p}|=k} B^{\mathbf{p}} \otimes Y^{\mathbf{p}} \otimes A_{(\mathbf{p})}\right)\right\| \leq 1 .
$$

(v) If $\left(T_{1}, \ldots, T_{n}\right) \in\left[B(\mathcal{K})^{n}\right]_{1 / 3}$ and $T_{1}, \ldots, T_{n}$ are commuting operators, then

$$
\sum_{k=0}^{\infty}\left\|\sum_{\mathbf{p} \in \mathbb{Z}_{+}^{n},|\mathbf{p}|=k} T^{\mathbf{p}} \otimes A_{(\mathbf{p})}\right\| \leq\left\|A_{(0)}\right\|+\left\|I-A_{(0)}\right\| .
$$

Proof. Notice that

$$
f_{\mathrm{sym}}\left(r S_{1}, \ldots, r S_{n}\right)=\sum_{k=0}^{\infty} \sum_{|\alpha|=k} r^{|\alpha|} S_{\alpha} \otimes C_{(\alpha)},
$$

where $C_{(0)}:=A_{(0)}$ and $C_{(\alpha)}:=\frac{\mathbf{p} !}{|\mathbf{p}| !} A_{(\mathbf{p})}$ for $\mathbf{p} \in \mathbb{Z}_{+}^{n}, \mathbf{p} \neq(0, \ldots, 0)$, and $\alpha \in \Lambda_{\mathbf{p}}$. We remark that condition (4.1) implies that $f_{\text {sym }}\left(r S_{1}, \ldots, r S_{n}\right)$ is norm convergent for each $r \in[0,1)$. It is clear that, for each $k=1,2, \ldots$, we have

$$
\begin{aligned}
\sum_{|\alpha|=k} C_{(\alpha)}^{*} C_{(\alpha)} & =\sum_{\mathbf{p} \in \mathbb{Z}_{+}^{n},|\mathbf{p}|=k}\left(\sum_{\alpha \in \Lambda_{\mathbf{p}}} C_{(\alpha)}^{*} C_{(\alpha)}\right) \\
& =\sum_{\mathbf{p} \in \mathbb{Z}_{+}^{n},|\mathbf{p}|=k} \frac{\mathbf{p} !}{|\mathbf{p}| !} A_{(\mathbf{p})}^{*} A_{(\mathbf{p})} .
\end{aligned}
$$

Now apply Theorem 2.5 to the holomorphic function

$$
F\left(X_{1}, \ldots, X_{n}\right):=f_{\text {sym }}\left(X_{1}, \ldots, X_{n}\right),
$$

$\left(X_{1}, \ldots, X_{n}\right) \in\left[B(\mathcal{X})^{n}\right]_{<1}$, and notice that part (i) and (ii) follow from part (ii) and part (vi) of Theorem 2.5. To prove part (iii), define the sequence $\left\{Z_{(\alpha)}\right\}_{\alpha \in \mathbb{F}_{n}^{+}} \subset$ $B(\mathcal{K})$ by $Z_{(0)}:=Y_{(0)}$ and $Z_{(\alpha)}:=Y_{(\mathbf{p})}$, where $\mathbf{p} \in \mathbb{Z}_{+}^{n}, \mathbf{p} \neq(0, \ldots, 0)$, and $\alpha \in \Lambda_{\mathbf{p}}$. Notice also that

$$
\sum_{k=0}^{\infty}\left\|\sum_{|\alpha|=k} Z_{(\alpha)}^{*} Z_{(\alpha)}\right\|^{1 / 2}=\sum_{k=0}^{\infty}\left\|\sum_{\mathbf{p} \in \mathbb{Z}_{+}^{n},|\mathbf{p}|=k} \frac{|\mathbf{p}| !}{\mathbf{p} !} Y_{(\mathbf{p})}^{*} Y_{(\mathbf{p})}\right\|^{1 / 2} \leq \frac{1}{2} .
$$


Using Theorem 2.4 part (ii) when $F\left(X_{1}, \ldots, X_{n}\right)=f_{\text {sym }}\left(X_{1}, \ldots, X_{n}\right)$, we deduce that

$$
\left\|\sum_{k=0}^{\infty} \sum_{|\alpha|=k} S_{\alpha} \otimes C_{(\alpha)} \otimes Z_{(\alpha)}\right\| \leq 1,
$$

where the series converges in the norm topology. Again taking the compression to the symmetric Fock space and noticing that

$$
\begin{aligned}
\sum_{k=0}^{\infty} \sum_{|\alpha|=k} B_{\alpha} \otimes C_{(\alpha)} \otimes Z_{(\alpha)} & =\sum_{\mathbf{p} \in \mathbb{Z}_{+}^{n},|\mathbf{p}|=k}\left(\sum_{\alpha \in \Lambda_{\mathbf{p}}} \frac{\mathbf{p} !}{|\mathbf{p}| !} B^{\mathbf{p}} \otimes A_{\mathbf{p}} \otimes Y_{\mathbf{p}}\right) \\
& =\sum_{\mathbf{p} \in \mathbb{Z}_{+}^{n},|\mathbf{p}|=k} B^{\mathbf{p}} \otimes A_{\mathbf{p}} \otimes Y_{\mathbf{p}},
\end{aligned}
$$

we conclude the proof of part (iii) of the theorem.

Now, applying Theorem 2.5 part (i) to $f_{\text {sym }}\left(X_{1}, \ldots, X_{n}\right)$, we obtain

$$
\left\|\sum_{k=0}^{\infty} \sum_{|\alpha|=k} S_{\alpha} \otimes Y_{\alpha} \otimes C_{(\alpha)}\right\| \leq 1 .
$$

Again taking the compression to the symmetric Fock space, we deduce (iv). To prove part (v), we apply Theorem 2.6 to $f_{\text {sym }}\left(X_{1}, \ldots, X_{n}\right)$ and deduce the inequality

$$
\sum_{k=0}^{\infty}\left\|\sum_{|\alpha|=k} T_{\alpha} \otimes C_{(\alpha)}\right\| \leq\left\|C_{(0)}\right\|+\left\|I-C_{(0)}\right\| .
$$

On the other hand, since $T_{1}, \ldots, T_{n}$ are commuting, we deduce that

$$
\sum_{k=0}^{\infty}\left\|\sum_{|\alpha|=k} T_{\alpha} \otimes C_{(\alpha)}\right\|=\sum_{k=0}^{\infty}\left\|\sum_{\mathbf{p} \in \mathbb{Z}_{+}^{n},|\mathbf{p}|=k} T^{\mathbf{p}} \otimes A_{(\mathbf{p})}\right\| .
$$

This completes the proof.

Corollary 4.2. Let $f\left(\lambda_{1}, \ldots, \lambda_{n}\right):=\sum_{\mathbf{p} \in \mathbb{Z}_{+}^{n}} \lambda^{\mathbf{p}} a_{\mathbf{p}}, a_{\mathbf{p}} \in \mathbb{C}$, be an analytic function on $\mathbb{B}_{n}$ such that condition (4.1) holds, $f(0) \geq 0$, and

$$
\operatorname{Re} f_{\text {sym }}\left(r S_{1}, \ldots, r S_{n}\right) \leq I \quad \text { for } 0 \leq r<1 \text {. }
$$

Then

$$
\sum_{k=0}^{\infty}\left|\sum_{\mathbf{p} \in \mathbb{Z}_{+}^{n},|\mathbf{p}|=k} \lambda^{\mathbf{p}}\right| a_{\mathbf{p}}|| \leq 1
$$

for any $\lambda:=\left(\lambda_{1}, \ldots, \lambda_{n}\right) \in \mathbb{B}_{n}$ with $\|\lambda\|_{2} \leq \frac{1}{3}$.

Theorem 2.10 can be used to obtain a version of Theorem 4.1 for operator-valued polynomias in $\mathbb{B}_{n}$. Here is a scalar version.

Corollary 4.3. Let $m=2,3, \ldots$, and let

$$
p\left(\lambda_{1}, \ldots, \lambda_{n}\right):=\sum_{\mathbf{p} \in \mathbb{Z}_{+}^{n},|\mathbf{p}| \leq m-1} \lambda^{\mathbf{p}} a_{\mathbf{p}}, \quad a_{\mathbf{p}} \in \mathbb{C},
$$


be an analytic polynomial on $\mathbb{B}_{n}$, such that $p(0) \geq 0$ and $\operatorname{Re} p_{\mathrm{sym}}\left(S_{1}, \ldots, S_{n}\right) \leq I$. Then

$$
\sum_{k=0}^{m-1}\left|\sum_{\mathbf{p} \in \mathbb{Z}_{+}^{n},|\mathbf{p}|=k} \lambda^{\mathbf{p}}\right| a_{\mathbf{p}}|| \leq 1
$$

for any $\lambda:=\left(\lambda_{1}, \ldots, \lambda_{n}\right) \in \mathbb{B}_{n}$ with $\|\lambda\|_{2} \leq t_{m}$, where $t_{m}$ is the solution of equation (0.1).

Denote by $H_{\mathrm{sym}}^{\infty}\left(\mathbb{B}_{n}\right)$ the set of all analytic functions on $\mathbb{B}_{n}$ with scalar coefficients

$$
f\left(\lambda_{1}, \ldots, \lambda_{n}\right):=\sum_{\mathbf{p} \in \mathbb{Z}_{+}^{\mathbf{n}}} \lambda^{\mathbf{p}} a_{\mathbf{p}}, \quad a_{\mathbf{p}} \in \mathbb{C},
$$

such that

$$
\limsup _{k \rightarrow \infty}\left(\sum_{\mathbf{p} \in \mathbb{Z}_{+}^{n},|\mathbf{p}|=k} \frac{|\mathbf{p}| !}{\mathbf{p} !}\left|a_{\mathbf{p}}\right|^{2}\right)^{1 / 2 k} \leq 1
$$

and

$$
\|f\|_{\text {sym }}:=\sup _{0 \leq r<1}\left\|f_{\text {sym }}\left(r S_{1}, \ldots, r S_{n}\right)\right\|<\infty .
$$

According to [24], $H_{\mathrm{sym}}^{\infty}\left(\mathbb{B}_{n}\right)$ is a Banach space with respect to the the norm $\|\cdot\|_{\text {sym }}$, which contains all analytic polynomials on $\mathbb{B}_{n}$.

Corollary 4.4. Let $m=2,3, \ldots, \infty$, and let

$$
f\left(\lambda_{1}, \ldots, \lambda_{n}\right):=\sum_{\mathbf{p} \in \mathbb{Z}_{+}^{n},|\mathbf{p}| \leq m-1} \lambda^{\mathbf{p}} a_{\mathbf{p}}, \quad a_{\mathbf{p}} \in \mathbb{C},
$$

be an analytic function in $H_{\mathrm{sym}}^{\infty}\left(\mathbb{B}_{n}\right)$. Then

$$
\sum_{k=0}^{m-1}\left|\sum_{\mathbf{p} \in \mathbb{Z}_{+}^{n},|\mathbf{p}|=k} \lambda^{\mathbf{p}}\right| a_{\mathbf{p}}|| \leq\|f\|_{\text {sym }}
$$

for any $\lambda:=\left(\lambda_{1}, \ldots, \lambda_{n}\right) \in \mathbb{B}_{n}$ with $\|\lambda\|_{2} \leq t_{m}$, where $t_{m}$ is the solution of equation (0.1) if $m<\infty$ and $t_{\infty}=\frac{1}{3}$.

\section{REFERENCES}

[1] L. Aizenberg, Multidimensional analogues of Bohr's theorem on power series, Proc. Amer. Math. Soc. (128) 2000, 1147-1155. MR1636918 (2000i:32001)

[2] A. Arias and G. Popescu, Noncommutative interpolation and Poisson transforms, Israel J. Math. (115) 2000, 205-234. MR1749679(2001i:47021)

[3] W.B. Arveson, Subalgebras of $C^{*}$-algebras III: Multivariable operator theory, Acta Math. (181) 1998, 159-228. MR 1668582 (2000e:47013)

[4] H.P. Boas and D. Khavinson, Bohr's power series theorem in several variables, Proc. Amer. Math. Soc. (125) 1997, 2975-2979. MR 1443371 (98i:32002)

[5] H. Bohr, A theorem concerning power series, Proc. London Math. Soc. (2)13 1914, 1-5.

[6] S. Dineen and R.M. Timoney, On a problem of H. Bohr, Bull. Soc. Roy. Sci. Liege (60) 1991, 401-404. MR 1162791 (93e:46050)

[7] P.G. Dixon, Banach algebras satisfying the non-unital von Neumann inequality, Bull. London Math. Soc. (27) 1995, 359-362. MR1335287(96e:46061)

[8] L. Fejér, Über trigonometrische Polynome, J. Reine Angew. Math. (146) 1916, 53-82.

[9] E.v. Egerváry and O. Szász, Einige Extremalprobleme im Bereiche der trigonometrischen Polynome, Math. Zeitschrift (27) 1928, 641-652. MR1544931 
[10] U. Haagerup and P. de la Harpe, The numerical radius of a nilpotent operator on a Hilbert space, Proc. Amer. Math. (115) 1992, 371-379. MR.1072339 (92i:47002)

[11] V.I. Paulsen, Completely Bounded Maps and Dilations, Pitman Research Notes in Mathematics, Vol.146, New York, 1986. MR0868472 (88h:46111)

[12] V.I. Paulsen, G. Popescu, and D. Singh, On Bohr's inequality, Proc. London Math. Soc. 85 (2002), 493-512. MR1912059 (2003h:47025)

[13] V.I. Paulsen and D. Singh, Extensions of Bohr's inequality, preprint.

[14] G. Pisier, Similarity Problems and Completely Bounded Maps, Springer Lect. Notes Math., Vol.1618, Springer-Verlag, New York, 1995. MR1441076 (98d:47002)

[15] G. Popescu, Characteristic functions for infinite sequences of noncommuting operators, J. Operator Theory (22) 1989, 51-71. MR1026074 (91m:47012)

[16] G. Popescu, Multi-analytic operators and some factorization theorems, Indiana Univ. Math. J. (38) 1989, 693-710. MR.1017331 (90k:47019)

[17] G. Popescu, Von Neumann inequality for $\left(B(H)^{n}\right)_{1}$, Math. Scand. (68) 1991, 292-304. MR:1129595 (92k:47073)

[18] G. Popescu, Functional calculus for noncommuting operators, Michigan Math. J. (42) 1995, 345-356. MR.1342494 (96k:47025)

[19] G. Popescu, Multi-analytic operators on Fock spaces, Math. Ann. (303) 1995, 31-46. MR1348353 (96k:47049)

[20] G. Popescu, Positive-definite functions on free semigroups, Canad. J. Math. (48) 1996, no. 4, 887-896. MR1407612 (97m:47011)

[21] G. Popescu, Noncommutative disc algebras and their representations, Proc. Amer. Math. Soc. (124) 1996, 2137-2148. MR.1343719 (96k:47077)

[22] G. Popescu, Poisson transforms on some $C^{*}$-algebras generated by isometries, J. Funct. Anal. (161) 1999, 27-61. MR.1670202 (2000m:46117)

[23] G. Popescu, Unitary invariants in multivariable operator theory, preprint 2004.

[24] G. Popescu, Free holomorphic functions on the unit ball of $B(\mathcal{H})^{n}$, J. Funct. Anal. (241) 2006, 268-333. MR2264252

[25] S. Sidon, Uber einen Satz von Herrn Bohr, Math. Z. (26) 1927, 731-732. MR1544888

[26] M. Tomic, Sur un theoreme de H. Bohr, Math. Scand. (11) 1962, 103-106. MR0176040 $(31: 316)$

[27] W.F. Stinespring, Positive functions on $C^{*}$-algebras, Proc. Amer. Math. Soc. (6) 1955, 211216. MR0069403 (16:1033b)

[28] J. von Neumann, Eine Spectraltheorie für allgemeine Operatoren eines unitären Raumes, Math. Nachr. (4) 1951, 258-281. MR0043386(13:254a)

Department of Mathematics, The University of Texas at San Antonio, San Antonio, TEXAs 78249

E-mail address: gelu.popescu@utsa.edu 